Draft Version November 17, 2018

Preprint typeset using $\mathrm{IAT}_{\mathrm{E}} \mathrm{X}$ style emulateapj v. 5/2/11

\title{
ON THE MECHANISM OF HARD X-RAY EMISSION FROM MAGNETARS
}

\author{
ANDREi M. BELOBOROdOV \\ Physics Department and Columbia Astrophysics Laboratory, Columbia University, 538 West 120th Street New York, NY 10027; \\ amb@phys.columbia.edu \\ Draft version November 17, 2018
}

\begin{abstract}
Persistent activity of magnetars is associated with electric discharge that continually injects relativistic particles into the magnetosphere. Large active magnetic loops around magnetars must be filled with outflowing particles that interact with radiation via resonant scattering and spawn electronpositron pairs. The outflow energy is processed into copious $e^{ \pm}$until the plasma enters outer parts of the loop where the magnetic field is reduced below $10^{13} \mathrm{G}$. In the outer zone, photons scattered by the outflow do not convert to $e^{ \pm}$pairs and the outflow radiates its energy away. The escaping radiation forms a distinct hard X-ray peak in the magnetar spectrum. It has the following features: (1) Its luminosity $L=10^{35}-10^{36} \mathrm{erg} \mathrm{s}^{-1}$ can easily exceed the thermal luminosity from the magnetar surface. (2) Its spectrum extends from $10 \mathrm{keV}$ to the $\mathrm{MeV}$ band with a hard spectral slope, which depends on the object inclination to the line of sight. (3) The anisotropic hard X-ray emission exhibits strong pulsations as the magnetar spins. (4) The emission spectrum typically peaks around $1 \mathrm{MeV}$, but the peak position significantly oscillates with the spin period. (5) The emission is dominated by the extraordinary polarization mode at photon energies below $\sim 1 \mathrm{MeV}$. (6) The decelerated pairs accumulate and annihilate at the top of the magnetic loop, and emit the 511-keV line with luminosity $L_{\mathrm{ann}} \sim 0.1 L$. Features (1)-(3) agree with available data; (4)-(6) can be tested by future observations. Subject headings: plasmas - stars: magnetic fields, neutron - X-rays
\end{abstract}

\section{INTRODUCTION}

Besides spectacular outbursts, magnetars produce persistent or decaying X-ray emission with luminosity $L \sim$ $10^{34}-10^{36} \mathrm{erg} \mathrm{s}^{-1}$. Two peaks are observed in their $\mathrm{X}$-ray spectra, with comparable luminosities. The first peak is near $1 \mathrm{keV}$; it is associated with thermal emission from the neutron star surface. The second peak is above $100 \mathrm{keV}$. Its low-energy slope (between 10 and $100 \mathrm{keV}$ ) was observed in 7 magnetar 1 (Kuiper et al. 2008; Enoto et al. 2010), with a typical photon index $\Gamma \sim 1-1.5$. The emission shows pulsations with the rotation period; the pulsed fraction approaches $100 \%$ at high energies.

The present paper focuses on the mechanism of the hard X-ray component. The puzzle of hard X-ray emission was previously discussed in several works (Thompson \& Beloborodov 2005; Heyl \& Hernquist 2005; Beloborodov \& Thompson 2007; Baring \& Harding 2007; Lyubarsky \& Eichler 2008). In general, nonthermal luminosity from an isolated neutron star must be fed by some form of energy release in its magnetosphere. In magnetars, this process occurs in the closed magnetosphere via electric discharge (Beloborodov \& Thompson 2007). The created relativistic particles move along the magnetic field lines and may radiate their kinetic energy in two ways:

1. Particles moving toward the neutron star will hit its surface and deposit their energy in a dense layer, which can radiate bremsstrahlung photons.

2. Particles can radiate their energy in the magnetosphere by scattering photons around the star.

1 Non-detections of similar hard X-ray emission in other magnetars are inconclusive because of insufficient sensitivity (W. Hermsen, private communication).
We consider in this paper magnetic loops that extend to radii $r \gtrsim 5 R$, where $R$ is the radius of the neutron star (Section 2.1). We assume that relativistic particles are injected in the low parts of the loops where the magnetic field is very strong, $B \gtrsim B_{Q}=m_{e}^{2} c^{3} / \hbar e=4.4 \times 10^{13} \mathrm{G}$. As explained below, the particles cannot immediately radiate their energy via scattering. Instead, the energy is adiabatically processed into a standard outflow along the magnetic field lines. This processing occurs via creation of secondary $e^{ \pm}$pairs whose number grows by a factor of $\sim 10^{2}$ as the flow moves away from the star toward the top of the magnetic loop (Figure 1). Pair loading ends where $B \approx 10^{13} \mathrm{G}$; at this point the outflow begins to lose energy to escaping photons. We find that the outflowing plasma radiates all its energy in the zone $3 \times 10^{11}<B<10^{13} \mathrm{G}$, in the upper parts of the loop. The released radiation is practically independent of the details of energy injection near the star because of the energy processing in the pair-loading zone $B>10^{13} \mathrm{G}$.

The paper is organized as follows. Section 2 describes the relativistic outflow, its formation and dynamics. It is approximated by a simple analytical model, which is confirmed by detailed numerical simulations in the accompanying paper (Beloborodov 2012, hereafter B12). Radiation emitted by the outflow is calculated in Section 3. It is found to have an extended hard spectrum between $10 \mathrm{keV}$ and $\sim 1 \mathrm{MeV}$ and form a pronounced hard X-ray peak in the magnetar spectrum. Section 4 explores the anisotropy and pulsations of radiation produced by this mechanism.

\section{RELATIVISTIC FLOW IN THE J-BUNDLE}

\subsection{Source of energy}

The radiative mechanism described in this paper is independent of the energy source as long as the source 


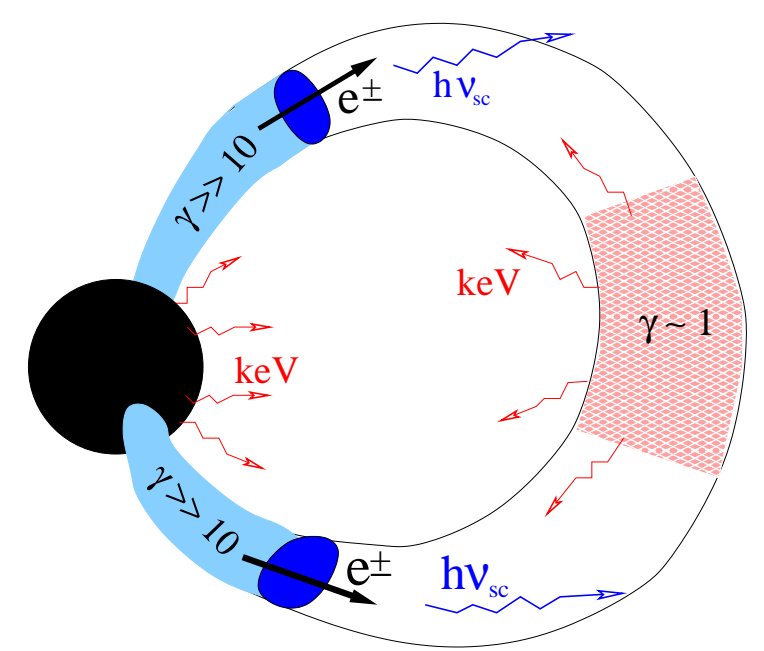

Figure 1. Sketch of an activated magnetic loop. Relativistic particles are injected near the star where $B>B_{Q}=4.4 \times 10^{13} \mathrm{G}$. Large $e^{ \pm}$multiplicity $\mathcal{M} \sim 100$ (Equation 11) develops in the adiabatic zone $B>10^{13} \mathrm{G}$ (shaded in blue). The outer part of the loop is in the radiative zone; here the scattered photons of energy $E=h \nu_{\mathrm{sc}}$ escape and form the hard X-ray spectrum that is calculated in Section 3. The outflow decelerates (and annihilates) at the top of the loop, shaded in pink; here it becomes very opaque to the thermal keV photons flowing from the star. Photons reflected from the pink region have the best chance to be upscattered by the relativistic outflow in the lower parts of the loop, and control its deceleration (Section 2.2).

generates relativistic particles near the magnetar where $B>10^{13}$ G. We here briefly describe the expected source.

Like the sun, magnetars are believed to have twisted magnetospheres that are deformed by surface motions (Thompson et al. 2000; 2002). Beloborodov (2009, hereafter B09) developed electrodynamic theory for the dissipative, twisted magnetosphere attached to a conducting sphere (neutron star). The theory predicts that the magnetic twist concentrates on field lines that extend far from the conducting star, forming an extended bundle of electric currents. This "j-bundle" is heated by ohmic dissipation and emits radiation. The currents are nearly force-free, $\mathbf{j} \times \mathbf{B}=0$, and sustained by a longitudinal voltage $\Phi$ along the magnetic field lines. Net current circulating through the magnetosphere, $I$, generates ohmic power $L=I \Phi$ that feeds the observed activity.

Similar ohmic heating occurs in ordinary pulsars, and it may be useful to compare them with magnetars. In ordinary pulsars, the magnetospheric twist is pumped at the light cylinder by the rotation of the star, and ohmic dissipation occurs on the open field lines. The electric current is then roughly given by $I \sim c \hat{\mu} / R_{\mathrm{lc}}^{2}$ (where $R_{\mathrm{lc}}$ is the light-cylinder radius and $\hat{\mu}$ is the magnetic dipole moment of the star), and voltage $\Phi$ can exceed $10^{12} \mathrm{~V}$. The dissipated power $I \Phi$ is always smaller than the spindown luminosity of the star. In magnetars, surface motions twist the closed magnetosphere. Then the electric current may be as large as $I \sim c \hat{\mu} / R^{2}$, where $R$ is the star radius. The characteristic voltage is $\Phi \sim 10^{9} \mathrm{~V}$ (Beloborodov \& Thompson 2007). The dissipated power $I \Phi$ is typically much larger than the spindown power.

Ohmic dissipation tends to gradually remove electric currents from the closed magnetosphere as described in B09. This process creates a growing "cavity" with vanished current density $j=0$. The currents have longest lifetimes on magnetic field lines with large apex radii $R_{\max } \gg R$. As a result, the magnetospheric activity becomes confined to the bundle of extended field lines. This theoretical picture agrees with observations (see Beloborodov 2011 for a review).

Consider magnetic field lines that extend sufficiently far from the star into the region where $B$ is smaller than a given value $B_{1}$. Luminosity that can be generated on these field lines may be estimated as follows. Approximating magnetic field by a dipole configuration with a dipole moment $\hat{\mu}$, one finds that the field lines reaching the region $B<B_{1}$ carry the magnetic flux $\digamma_{1}=2 \pi \hat{\mu} / R_{1}$ filling the hemisphere of radius $R_{1}=\left(\hat{\mu} / B_{1}\right)^{1 / 3}$. If the field lines are twisted with amplitude $\psi$, they must carry electric current (according to Stokes theorem, see B09 and Appendix C),

$$
I_{1} \approx \frac{c \psi \digamma_{1}}{8 \pi R_{1}}=\frac{c \hat{\mu} \psi}{4 R_{1}^{2}} .
$$

The twist amplitude $\psi$ is measured in radians. It has the meaning of relative azimuthal position of the northern and southern footpoints of the magnetospheric field line. The generated power is $L=I_{1} \Phi$, which yields

$$
\begin{array}{r}
L \approx 10^{36} \psi\left(\frac{\hat{\mu}}{10^{33} \mathrm{G} \mathrm{cm}^{2}}\right)^{1 / 3}\left(\frac{\Phi}{4 \times 10^{9} \mathrm{~V}}\right) \\
\times\left(\frac{B_{1}}{10^{12} \mathrm{G}}\right)^{2 / 3} \mathrm{erg} \mathrm{s}^{-1} .
\end{array}
$$

Strong twists tend to inflate the magnetosphere (Wolfson 1995; Parfrey et al. 2012a,b). As long as $\psi \lesssim 1$, this effect is modest (it scales as $\psi^{2}$ at $\psi<1$, see B09), and the poloidal field may be well approximated by a dipole configuration with dipole moment $\hat{\mu}$.

\subsection{Interaction of outflowing particles with radiation field}

Discharge with voltage $\Phi \sim 10^{9} \mathrm{~V}$ injects electrons (or positrons) with high Lorentz factors $\gamma \sim e \Phi / m_{e} c^{2} \sim 10^{3}$. The relativistic plasma created near the star expands along the magnetic field and forms a relativistic outflow, resembling the outflow along open field lines in ordinary pulsars (however, here plasma moves along closed field lines and is trapped in the magnetic loops around the neutron star). Magnetars are hot and bright; their dense radiation exerts a strong drag on the magnetospheric particles and controls the outflow velocity. On the other hand, the plasma significantly changes the radiation field around the magnetar. The interaction of the flowing $e^{ \pm}$plasma and radiation may be described as a self-consistent radiative transfer problem. This problem is solved numerically in the accompanying paper (Beloborodov, in preparation, hereafter B12). The results can be summarized as follows.

The outflow interacts with radiation via resonant scattering; other processes turn out unimportant. Consider an outflowing electron (or positron) with Lorentz factor $\gamma=\left(1-\beta^{2}\right)^{-1 / 2}$, and a target photon of energy $E_{t}$ propagating at an angle $\vartheta$ with respect to the outflow direction. Resonant scattering can occur if the photon energy in the electron rest frame,

$$
\tilde{E}=\gamma(1-\beta \cos \vartheta) E_{t},
$$


matches Landau energy $\hbar \omega_{B}$,

$$
\tilde{E}=\hbar \omega_{B}=b m_{e} c^{2}, \quad b \equiv \frac{B}{B_{Q}} .
$$

The relativistic outflow in the low parts of magnetic loops interacts with thermal $\mathrm{keV}$ radiation that has been reflected by plasma trapped at the top of the magnetic loops (Figure 1). The outflow sees the reflected photons as main targets because they propagate at most favorable angles for resonant scattering (Appendix A).

Scattering of thermal keV radiation gives a huge energy boost to photons, on average by a factor $\sim \gamma^{2}$. The relativistic outflow experiences significant energy losses by scattering a tiny fraction of photons around the magnetar. The outflow adjusts so that its scattering rate is just enough for the self-consistent deceleration. In essence, the relativistic outflow moves fast enough to interact with photons of energy $E \sim 10 k T$ (the low-density exponential tail of the thermal spectrum), and slow enough to not interact with the main peak of the thermal spectrum $E \sim 3 k T$. This condition, together with Equation (44), determines that the scattering plasma moves with Lorentz factor

$$
\gamma_{\mathrm{sc}} \approx \frac{b m_{e} c^{2}}{10 k T}
$$

The self-regulation of Lorentz factor is a key feature of the outflow that allows one to calculate its emission spectrum (Section 3). This feature is illustrated in Figure 2 that shows the dynamics of a relativistic electron resonantly interacting with radiation. The electron was injected with Lorentz factor $\gamma=1000$ at $B=10 B_{Q}$ and the figure shows its trajectory in the $B-\gamma$ plane. As the electron moves outward along the magnetic field line, it scatters photons and loses energy. Each scattering event is a step down in $\gamma$ at fixed $\mathrm{B}$, and each free path between subsequent scatterings is a step down in $B$ at constant $\gamma$. In this figure, the mean free path $\ell(b)$ is plotted between scattering events; a full Monte-Carlo simulation will be shown in Section 3.

The average effect of scattering may be described as a drag force acting on the electron, $\mathcal{F}(B, \gamma)$. This force $\mathcal{F}$ is sensitive to the electron position in the $B-\gamma$ plane. As indicated in the figure, the drag is enormous above the electron trajectory and negligible below the trajectory. In essence, the electron surfs the steep slope of $\mathcal{F}(B, \gamma)$ so that it stays outside and around the strongdrag region of the $B-\gamma$ plane. The relevant dimensionless parameter measuring the drag effect is roughly given by (see Appendix A),

$$
D=\frac{r \mathcal{F}}{\gamma m_{e} c^{2}} \sim 10 \frac{r}{R} \frac{y^{2}}{e^{y}}, \quad y=\frac{\hbar \omega_{B}}{\gamma\left(1-\beta \cos \vartheta_{\max }\right) k T},
$$

where $\vartheta_{\max }$ is the maximum angle of target photons with respect to the electron velocity; $\cos \vartheta_{\max }=-0.5$ is assumed in Figure 2. The electron surfs with $D \sim$ $|d \ln B / d \ln r| \sim 3$, which corresponds to $y=6-7$ and $\gamma \approx 100 b(k T / 0.5 \mathrm{keV})^{-1}$. This condition defines a line in the $B-\gamma$ plane that quite accurately describes the electron trajectory, confirming Equation (5).

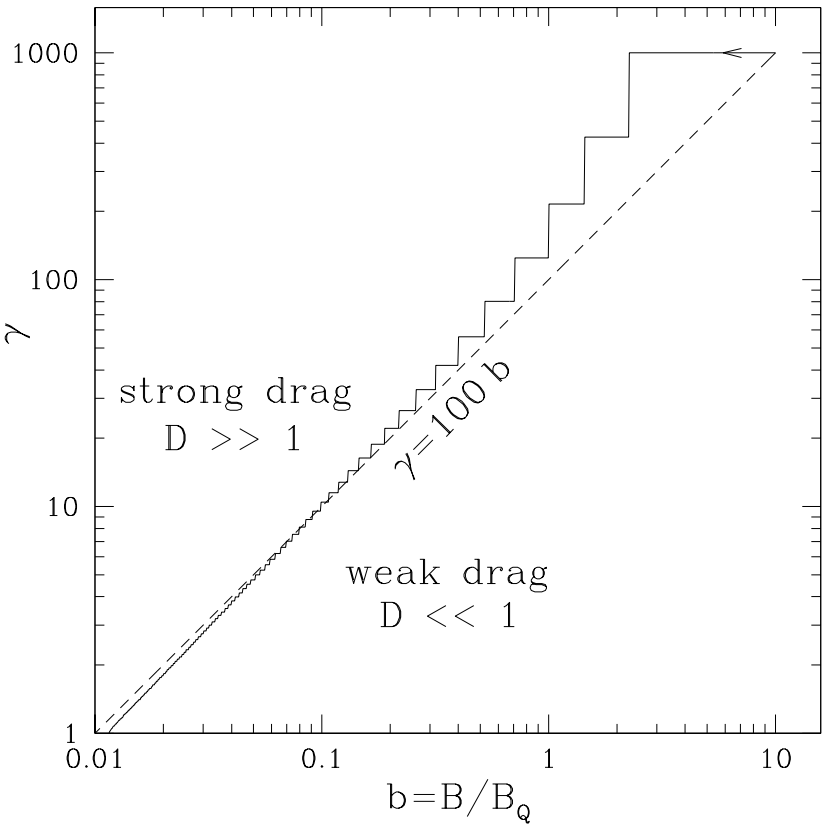

Figure 2. Deceleration of a relativistic electron moving along the magnetic field line away from the star and interacting with ambient radiation field of temperature $k T \approx 0.5 \mathrm{keV}$ (see text). Each step down in $\gamma$ is a result of one scattering.

\subsection{Two zones of the outflow deceleration \\ 2.3.1. Pair loading zone}

The relativistic outflow created in the ultra-strong magnetic field must decelerate as it moves to weaker fields, according to Equation (5). However, it is initially unable to radiate its energy away, because the scattered photons quickly convert to $e^{ \pm}$pairs that join the outflow. The deceleration occurs because the flow kinetic energy is shared by more particles.

The mean expectation for the energy of a resonantly scattered photon is given by (see Equation A2)

$$
E=\gamma_{\mathrm{sc}} b m_{e} c^{2} q(b), \quad q=\frac{1}{b}\left(1-\frac{1}{\sqrt{1+2 b}}\right),
$$

which states that the photon energy in the electron frame, $\tilde{E}=b m_{e} c^{2}$ (Equation 4), is blueshifted in the lab frame by the typical Doppler factor $\sim \gamma_{\mathrm{sc}}$. The factor $q<1$ is a correction due to electron recoil in scattering. In weak fields $q \approx 1$, and in strong fields $(b \gg 1) q \approx b^{-1} \ll 1$, consistent with the evident requirement $E<\gamma_{\mathrm{sc}} m_{e} c^{2}$. Combining Equation (7) with Equation (5), one finds that photons capable of converting to $e^{ \pm}$pairs, $E>2 m_{e} c^{2}$, are generated in the region of rather strong magnetic field $b>b_{0} \sim 1 / 4$. A more accurate formula for $b_{0}$ is given in Appendix B.

Photons scattered in the zone $b>b_{0}$ do not escape. Their fate depends on the polarization state: || photons directly convert to $e^{ \pm}$, and $\perp$ photons split in two daughter $\|$ photons (see Appendix A for the definition of $\|$ and $\perp$ polarization states). Conversion occurs immediately once the $\|$ photon satisfies the threshold condition,

$$
E>E_{\mathrm{thr}}=\frac{2 m_{e} c^{2}}{\sin \vartheta}
$$


where $\vartheta$ is the photon angle with respect to the magnetic field. The angle is initially small after scattering, $\vartheta \sim$ $\gamma_{\mathrm{sc}}^{-1}$, however it quickly grows as the photon propagates through the curved magnetic field. Therefore, almost all $\|$ photons of energy $E \gg 2 m_{e} c^{2}$ produced in the zone $b>b_{0}$ are quickly absorbed. This fact is observed in our numerical simulations and discussed in Appendix B (cf. Medin \& Lai [2010], where a similar problem was studied for the polar cap near the magnetic axis).

In summary, the outflow radiation in the zone $b>b_{0} \sim$ $1 / 4$ is quickly absorbed and transformed into secondary $e^{ \pm}$pairs. This has a deceleration effect on the outflow, as the energy per particle is reduced (while the net energy of particles is conserved, since almost no photons escape this zone).

\subsubsection{Radiative zone}

As the outflow approaches $b_{0}$, the scattered photons begin to escape. In the region $b<0.1$ all scattered photons avoid conversion and splitting, and escape the magnetosphere, forming the hard X-ray component of the magnetar spectrum. As the outflow reaches the region $b \sim 0.01, \gamma_{\mathrm{sc}}$ decreases to a mildly relativistic value $\sim 1$ (see Equation 5), i.e. essentially all its energy has been radiated away.

We conclude that the relativistic outflow radiates almost all its energy in a well-defined region of magnetic field $0.01 \lesssim b \lesssim 1 / 4$, regardless of the details of magnetospheric configuration - the same conclusion is valid for a multipolar twisted magnetic field. Section 3 will describe a simple method to calculate the produced radiation.

\subsection{Multiplicity of $e^{ \pm}$pairs}

Let $2 \dot{N}$ be the number flux of $e^{ \pm}$outflowing along both legs of the magnetic loop toward its top. Multiplicity is defined by

$$
\mathcal{M}=\frac{e \dot{N}}{I},
$$

where $I$ is the electric current circulating in the magnetic loop. $\dot{N}$ grows in the pair-loading zone $b>b_{0}$ to its final value that can be evaluated at $b \approx b_{0}$ using

$$
2 \dot{N} \bar{\gamma}\left(b_{0}\right) m_{e} c^{2} \approx L=\Phi I
$$

Here $\bar{\gamma}\left(b_{0}\right)$ is the average outflow Lorentz factor at $b \approx b_{0}$. It is close to $\gamma_{\mathrm{sc}}$ given in Equation (5); $; \bar{\gamma} \lesssim \gamma_{\mathrm{sc}}$ is expected if the particle momentum distribution has a significant width (see Section 2.5). Then one finds,

$$
\mathcal{M} \approx \frac{e \Phi}{2 \bar{\gamma}\left(b_{0}\right) m_{e} c^{2}} \sim 10^{2}
$$

where we used the estimate $\bar{\gamma}\left(b_{0}\right) \lesssim \gamma_{\mathrm{sc}}\left(b_{0}\right) \sim 20$.

The large multiplicity of created pairs implies that the outflow can easily conduct the electric current $I$ required by the twisted magnetic field and screen electric fields. Therefore, no "gaps" with accelerating electric field are expected in the outer magnetosphere.

\subsection{Momentum distribution and collective effects}

A simplest model of $e^{ \pm}$outflow would assume that all particles have the Lorentz factor given by Equation (5). In fact, the local distribution of Lorentz factor cannot be a delta-function $\delta\left(\gamma-\gamma_{\mathrm{sc}}\right)$ - the real distribution must be broad. It is influenced by three effects:

(1) Cold, single-fluid flow would be unable to carry the required electric current. An electric field is induced in the magnetosphere to separate the average velocities of the positive and negative charges, so that the electric current is organized in the (nearly neutral) outflowing plasma (B12). It enforces a minimum width of the momentum distribution of $e^{ \pm}$.

(2) The cascade in the pair-loading zone injects particles with a broad range of momenta (see Section 3.2 below). (3) The momentum distribution of created particles $f_{e}(p)$ is prone to two-stream instability. The instability tends to thermalize the distribution and reduce the gradients $d f_{e} / d p$ that are responsible for the instability.

Remarkably, it is possible to evaluate the outflow radiation even without knowing exactly how $f_{e}(p)$ is changed by collective electric fields. This is possible because the outflow with a broad distribution function still must scatter radiation with Lorentz factor $\gamma_{\mathrm{sc}}$ given by Equation (5), and the scattering rate is still dictated by how quickly the outflow must decelerate, keeping the particles at $\gamma \lesssim \gamma_{\text {sc }}$ and avoiding excessive drag. The surfing described in the end of Section 2.2 is still a valid description of the outflow dynamics. A large width of $f_{e}(p)$ only implies that at any given location a fraction of particles actually scatter radiation — those particles that have $\gamma \approx \gamma_{\mathrm{sc}}$ - while all other particles do not have sufficiently high Lorentz factors for resonant scattering. The energies of scattered photons $E_{\mathrm{sc}}$ and the net scattering rate are not much different from the single-fluid model. This fact is observed in the simulations of B12, where $f_{e}(p)$ is broad.

\section{CALCULATION OF HARD X-RAY EMISSION}

We build the emission model starting from the simple consideration of a single particle. Then we consider emission from the $e^{ \pm}$cascade that develops along the magnetic loop, without taking into account collective effects. Finally, we formulate an approximate model for the $e^{ \pm}$outflow with collective behavior, which will be used to calculate sample spectra in Section 4.

\subsection{Emission from one particle}

Consider the simplest case of one particle shown in Figure 2. The particle is injected with a high Lorentz factor in the low part of the magnetic loop and decelerates as it moves away from the star. What is the net spectrum of radiation emitted by the particle as it decelerates to $\gamma \sim 1$ ?

The Lorentz factor $\gamma$ decreases along the "scattering curve," $\gamma \approx \gamma_{\mathrm{sc}}$, which is given by Equation (5). It will be convenient to rewrite Equation (5) in the form,

$$
\gamma_{\mathrm{sc}}(b)=K b, \quad K \approx 10^{2}\left(\frac{k T}{0.5 \mathrm{keV}}\right)^{-1} .
$$

The particle energy is passed to high-energy photons that are generated by scattering thermal radiation. Each scattered photon has energy $\widetilde{E}=b m_{e} c^{2}$ in the particle rest frame, as the scattering is resonant ${ }^{2}$ Its blueshifted energy in the observer frame $E$ depends on the scattering

2 We consider the radiative zone where $b \ll 1$ and therefore neglect corrections due to electron recoil. 
angle. A simplest estimate for the emitted spectrum is obtained if we neglect the dispersion in scattering angles and take $E$ equal to its average value $\gamma \tilde{E}$,

$$
E=\gamma b m_{e} c^{2}
$$

Since the particle moves with $\gamma \approx K b$ (Figure 2), we can substitute $b=\gamma / K$ in Equation (13) and obtain the relation between the emitted photon energy $E$ and the particle energy $\gamma m_{e} c^{2}$,

$$
\gamma m_{e} c^{2}=\left(K m_{e} c^{2} E\right)^{1 / 2} \text {. }
$$

Energy $d W_{1}=d \gamma m_{e} c^{2}$ is radiated in photons of energy $E$, and the net emitted spectrum is given by

$$
\frac{d W_{1}}{d E}=\frac{d \gamma}{d E} m_{e} c^{2}=\frac{1}{2}\left(\frac{K m_{e} c^{2}}{E}\right)^{1 / 2} .
$$

Since $E \propto b^{2}$, the most energetic photons are emitted at the highest $b \approx b_{0}$, near the boundary of the radiative zone, with energies comparable to $1 \mathrm{MeV}$. As the particle moves to smaller magnetic fields $b$ and its Lorentz factor decreases, softer photons are produced, forming the spectrum $\propto E^{-1 / 2}$.

\subsection{Emission from $e^{ \pm}$cascade (no collective effects)}

Again consider one high-energy particle injected with a Lorentz factor $\gamma_{p}>100$ at $b>1$. Emission from the particle in the radiative zone $b<b_{0}$ was evaluated in Section 3.1. Before emitting the escaping radiation, the particle scatters photons in the zone $b>b_{0}$ and thus generates secondary $e^{ \pm}$pairs. The secondary particles also scatter photons, which can convert to more pairs. All $e^{ \pm}$ pairs generated in this cascade enter the radiative zone and eventually decelerate to $\gamma \sim 1$ as they outflow along the magnetic loop. What emission should be observed from the cascade?

In this section, we adopt the assumption that all particles flow freely and change their $\gamma$ only when they scatter a photon. This model ignores the interaction between particles via collective electric fields (Section 2.5). The opposite case of strong collective effects will be considered in Section 3.3.

The development of the cascade is shown in Figure 3; the details of the calculation are given in Appendices A and $\mathrm{B}$. Each created particle follows a track in the $B-\gamma$ plane similar to that in Figure 2, except that the secondary particles are injected with smaller energies. They start their tracks significantly below the scattering curve $\gamma_{\mathrm{sc}}=K b$ and have a long free path with $\gamma=$ const before reaching the curve; then the particles begin to frequently scatter radiation and surf along the curve as shown in Section 2.2.

The cascade develops in the zone of a strong magnetic field where the photon splitting rate is high (Appendix B). The scattered photon can be in one of the two polarization states, $\perp$ or $\|$. The energetic $\perp$ photon quickly splits in two daughter photons with $\|$ polarization, before it gets a chance to convert to $e^{ \pm}$. The $\|$ photons do not split; they convert to $e^{ \pm}$. The conversion occurs immediately after the propagating photon meets the threshold condition (8). Therefore, $e^{-}$and $e^{+}$are created with total energy $E_{ \pm} \approx E_{\mathrm{thr}}$, in the ground Landau state, and do not produce synchrotron emission.

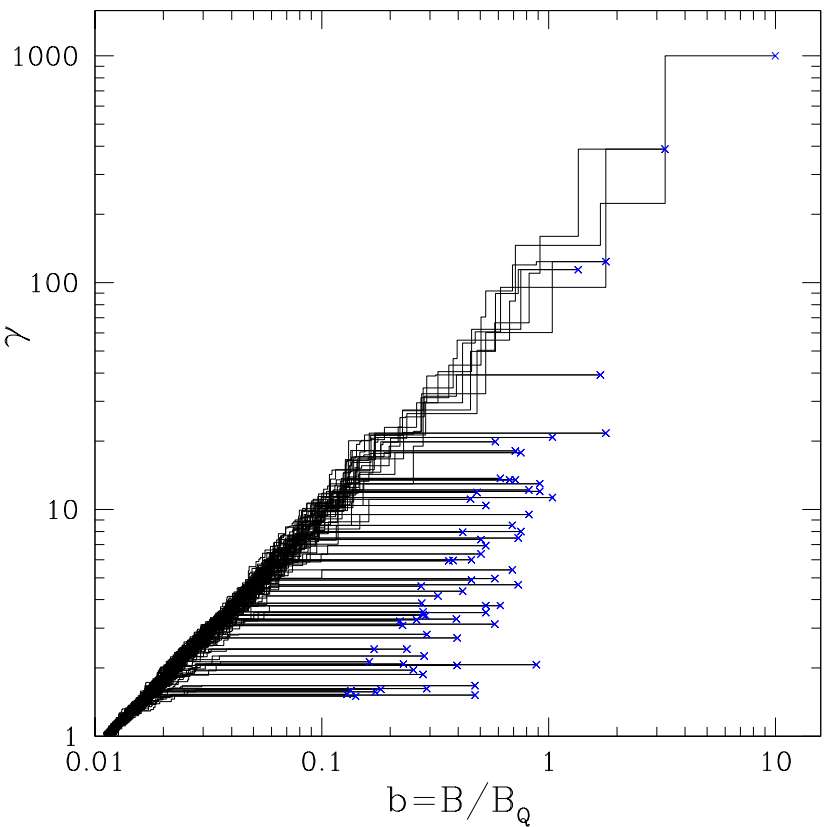

Figure 3. One random realization of $e^{ \pm}$cascade produced by one electron injected with Lorentz factor $\gamma_{p}=10^{3}$ at magnetic field $B=10 B_{Q} .136$ secondary particles are created in this cascade; the injection points for the primary particle and 68 secondary pairs are shown by blue crosses. After injection, each particle moves toward and then along the "scattering curve," which is approximately described by Equation (5).

Note that pair creation takes place in the region $b>0.1$ where photons should convert to positronium rather than free $e^{ \pm}$(Shabad \& Usov 1986). The binding energy of positronium atoms is $\tilde{E}_{b} \sim 0.1 \mathrm{keV}$, and they are easily unbound by photons of energy comparable to $\tilde{E}_{b}$ (in the atom rest frame), i.e. by UV photons of energy $\sim 0.1 \gamma^{-1} \mathrm{keV}$ in the lab frame, where $\gamma=E_{ \pm} / 2 m_{e} c^{2}$ is the Lorentz factor of the positronium atom. The rate of this reaction in a thermal radiation field was evaluated by Bhatia et al. (1988). In our problem, the positronium Lorentz factors are modest, and the resulting ionization rate is so high that essentially all $e^{ \pm}$pairs must be unbound. Even if a positronium atom reaches the scattering curve before ionization, $e^{+}$and $e^{-}$begin to resonantly interact with X-rays as if they were free particles (because $\hbar \omega_{B} \gg \tilde{E}_{b}$ ) and immediately become unbound due to recoil in scattering.

Our cascade simulation shows that the total number of secondary $e^{ \pm}$pairs (generated per one electron injected with Lorentz factor $\left.\gamma_{p}=10^{3}\right)$ is $N_{\text {inj }} \approx 10^{2}$, similar to estimate (11). The particle Lorentz factors at injection points (blue crosses in Figure 3) form a distribution $d N_{\text {inj }} / d \gamma$ that can be accurately calculated by simulating a large number $\mathcal{N}$ of random realizations of the cascade. We use $\mathcal{N}=10^{4}$ to accumulate sufficient statistics. The obtained distribution $d N_{\text {inj }} / d \ln \gamma$ is shown in Figure 4 . It is very well approximated by a simple power law,

$$
\frac{d N_{\text {inj }}}{d \ln \gamma}=A \gamma^{-q}, \quad \gamma>\gamma_{1} .
$$

Here $\gamma_{1}=E / 2 m_{e} c^{2} \sim 1-2$ corresponds to photons of minimum energy $E$ that are able to convert to $e^{ \pm}$; in our 


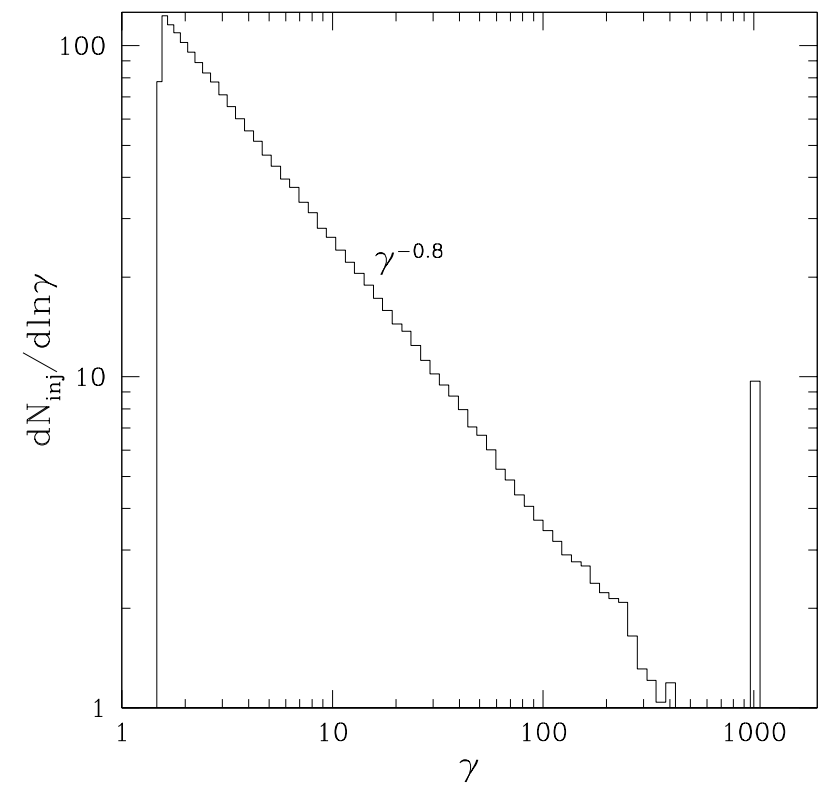

Figure 4. Injection Lorentz factor distribution of $e^{ \pm}$pairs in cascades generated by one particle with $\gamma_{p}=10^{3}$ (cf. blue crosses in Figure 3, which shows one realization of the cascade). The injection of the primary particle is shown by the peak at $\gamma=10^{3}$ (normalized to unity), and the broad smooth distribution shows the injection of secondary $e^{ \pm}$. Straight line shows the approximation given in Equation (16).

sample model $\gamma_{1}=1.5$. The slope of the distribution in Figure 4 is $q \approx 0.84$, and the corresponding normalization factor $A$ is given by $A=q \gamma_{1}^{q} N_{\mathrm{inj}} \approx 0.86 N_{\mathrm{inj}}$.

A cascade is characterized by its "pair yield" $Y$. It is defined as the mean expectation for the ratio of the total rest-mass energy of created pairs, $N_{\mathrm{inj}} m_{e} c^{2}$, to the energy of the primary particle $\gamma_{p} m_{e} c^{2}$. We find

$$
Y=\frac{N_{\text {inj }}}{\gamma_{p}}=0.15
$$

The same Monte-Carlo simulation gives the spectrum of radiation emitted by the cascade. We accumulate the statistics of all escaping photons (which avoid splitting and conversion) in $\mathcal{N}=10^{4}$ realizations of the cascade, and find their average spectrum. The result is shown in Figure 5. In this simulation, we assume that radiation is produced by a thin magnetic tube for which the highest energy of escaping photons $E_{\max }=3 m_{e} c^{2}$ (these photons are emitted at $b \approx b_{0} \approx 0.3$ ); therefore the obtained spectrum has a sharp break at $E \approx 1.5 \mathrm{MeV}$. We also performed more detailed simulations that track all photons as they propagate through the magnetosphere and check for absorption or splitting along their trajectories (Appendix B); this gave a similar break at $E_{\max }$ whose exact value (comparable to $3 m_{e} c^{2}$ ) depends on the radius of curvature of the magnetic tube.

A simple analytical estimate for the spectral slope below $1 \mathrm{MeV}$ can be derived as follows. Each injected particle begins to radiate when it reaches the scattering curve $\gamma=K b$. Each particle surfing along the curve emits the same standard spectrum $d W_{1} / d E$ given by Equation (15). Hence radiation produced at a given photon

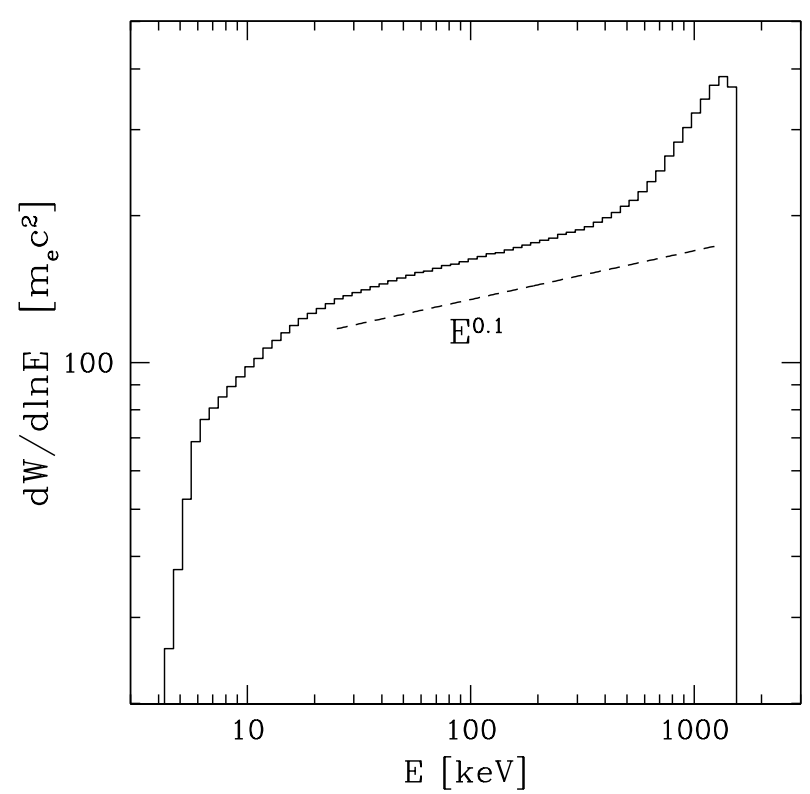

Figure 5. Average spectrum of escaping radiation from cascades generated by primary particles with Lorentz factor $\gamma_{p}=10^{3}$. The total emitted energy $W$ equals the injected energy $\gamma_{p} m_{e} c^{2}$. Dashed line shows the analytical estimate (Equation 20). Photon splitting creates an additional bump that is visible at $E \sim 1 \mathrm{MeV}$. The bump consists of photons with $\|$ polarization. Emission at lower energies $E \ll 1 \mathrm{MeV}$ is dominated by photons with $\perp$ polarization.

energy $E$ is given by

$$
\frac{d W}{d E}=\frac{d W_{1}}{d E} \int_{\gamma_{E}}^{\gamma_{p}} \frac{d N_{\mathrm{inj}}}{d \gamma} d \gamma, \quad \gamma_{E}=\left(\frac{K E}{m_{e} c^{2}}\right)^{1 / 2},
$$

where $\gamma_{E}$ is the Lorentz factor of particles that emit at energy $E$ (Equation [13). From Equations (18) and (16) we find

$$
\frac{d W}{d \ln E}=\frac{A}{2 q} m_{e} c^{2}\left(\frac{K E}{m_{e} c^{2}}\right)^{\frac{1-q}{2}}, \quad \frac{E}{m_{e} c^{2}}>\frac{\gamma_{1}^{2}}{K},
$$

which gives

$$
\frac{d W}{d \ln E} \approx 0.1 \gamma_{p} m_{e} c^{2}\left(\frac{K E}{m_{e} c^{2}}\right)^{0.1},
$$

where we used Equation (17), $A / q=N_{\text {inj }} \gamma_{1}^{q}$, and $q \approx 0.8$. This expression only describes radiation produced by scattering; it does not take into account photon splitting. It gives a reasonable approximation to the emitted spectrum at $E<m_{e} c^{2}$. The splitting of high-energy photons with the $\perp$ polarization, $\perp \rightarrow\|+\|$, creates additional photons that form a bump near $E \sim 1 \mathrm{MeV}$ (Figure 5).

The cascade model described above has one feature that makes the model questionable: the momentum distribution of $e^{ \pm}$develops two peaks and becomes unstable. We have calculated the distribution of $e^{ \pm}$created by the cascade and how it evolves with $b$ as the flow moves along the tube. The momentum distribution at three locations in the tube is shown in Figure 6. One can see the development of two peaks. The low-energy peak $(p \approx 1.5$ at $b=0.07)$ is formed by recently injected secondary particles. The high-energy peak $(p \approx 7$ at $b=0.07)$ is formed by older particles that were injected at higher $b$ and are 


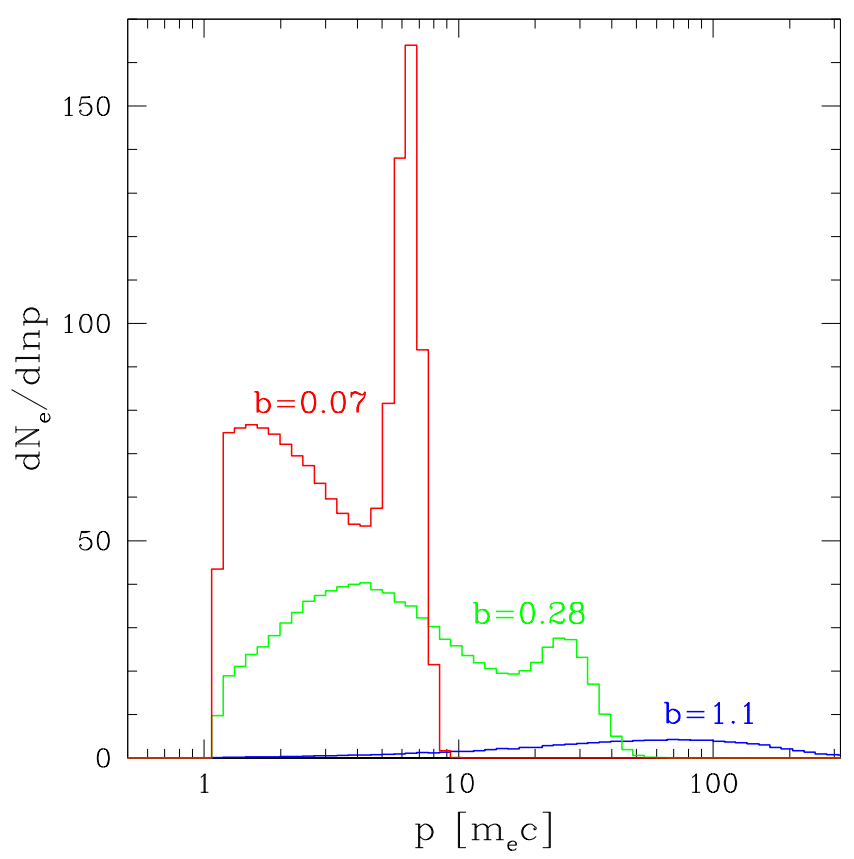

Figure 6. Momentum distribution of outflowing $e^{ \pm}$pairs created by the cascade, without taking into account collective effects. The distribution changes along the magnetic tube with decreasing $b=B / B_{Q}$ as more particles are created and the particles lose momentum to scattering.

now moving along the scattering curve (cf. Figure 3); its position evolves with $b$ according to $p \approx \gamma_{\mathrm{sc}} \approx 100 b$ (Equation 12).

The two-peak distribution is prone to plasma instability that excites Langmuir waves in the outflow. We conclude that the model without collective effects is unstable, and the excitation of collective electric fields is inevitable, which will change the distribution function. Below we will seek a simplest emission model for the $e^{ \pm}$ outflow with collective behavior.

\subsection{Emission from $e^{ \pm}$outflow with collective behavior}

At a given location in the active loop, only a small fraction of particles have $\gamma \approx \gamma_{\mathrm{sc}}$ that is high enough to resonantly scatter radiation; most particles have $\gamma<\gamma_{\mathrm{sc}}$ and do not interact with radiation. This fact is true in the absence of collective effects (see Figure 3) and remains true in collective models (cf. numerical simulations in B12). Since radiation is scattered by particles with known Lorentz factor $\gamma \approx \gamma_{\mathrm{sc}}$ (Equation [12), the details of $f_{e}(p)$ are not important for the produced emission. It turns out that it is sufficient to know the average Lorentz factor $\bar{\gamma}(b)$,

$$
\bar{\gamma}=\int\left(1+p^{2}\right)^{1 / 2} f_{e}(p) d p .
$$

Our model of $e^{ \pm}$outflow with collective behavior will avoid the complicated calculation of $f_{e}(p)$; instead, we focus on $\bar{\gamma}$ and use the following approximation,

$$
\bar{\gamma} \propto \gamma_{\mathrm{sc}},
$$

where $\gamma_{\mathrm{sc}}$ is given by Equation (12). In essence, $\bar{\gamma}(b)$ is kept somewhat below $\gamma_{\mathrm{sc}}(b)$ - the function $\gamma_{\mathrm{sc}}(b)$ shows how the outflow must decelerate as it enters weaker magnetic fields. As an example of collective behavior, one can picture an outflow whose distribution function maintains a constant shape, which shifts to lower $\gamma$ as the outflow moves to weaker magnetic fields. Approximation (22) greatly simplifies the calculation of generated radiation. Remarkably, the details of the cascade, its multiplicity, and the coefficient of proportionality in Equation (22) turn out unimportant - they drop out from the calculation as will be seen below.

Consider a thin active magnetic flux tube. We wish to know how its luminosity $L$ is distributed over $b$ along the tube, as this will determine the spectrum of produced radiation. We will use the simple fact that the power emitted by the tube element of length $d l$ equals the power lost by the outflow, i.e. the emitted power is determined by the deceleration law.

In the radiative zone, where no new $e^{ \pm}$pairs are created, the particle number flux $\dot{N}$ is constant along the tube up to its summit, where particles annihilate. The outflow power $2 \dot{N} \bar{\gamma} m_{e} c^{2}$ (factor of 2 for two legs of the tube) decreases proportionally to $\bar{\gamma}$. The lost power transforms to radiation, and hence the produced luminosity is distributed over $b$ according to

$$
\frac{d L}{d b}=2 \dot{N} \frac{d \bar{\gamma}}{d b} m_{e} c^{2}=\text { const. }
$$

Here $b=B / B_{Q}$ is used as a coordinate along the magnetic flux tube; expression on the left side is constant along the tube because $\bar{\gamma}$ is proportional to $\gamma_{\mathrm{sc}}$ (Equation 22) and $\gamma_{\mathrm{sc}}$ is proportional to $b$ (Equation 12), so $d \bar{\gamma} / d b=$ const. The total power $L$ (emitted in the radiative zone $\left.b<b_{0}\right)$ equals $(d L / d b) b_{0}$, which gives

$$
\frac{d L}{d b}=\frac{L}{b_{0}} .
$$

Equations (12) and (24) lead to a complete description of the emitted radiation, as at every $b$ they determine the Lorentz factor of the emitting particles $\gamma_{\mathrm{sc}}$ and the emission rate. Consider first the net spectrum of radiation emitted by the tube in all directions. Similarly to Section 2.1, a simplest estimate is obtained if we neglect the dispersion in scattering angles and take photon energy $E$ equal to its mean expectation $\gamma_{\mathrm{sc}} b m_{e} c^{2}$,

$$
E=K m_{e} c^{2} b^{2} .
$$

Then one finds $d b=(1 / 2)\left(K m_{e} c^{2} E\right)^{-1 / 2} d E$, and substitution to Equation (24) gives the spectrum emitted by the tube,

$$
\frac{d L}{d E}=\frac{L}{2 b_{0}\left(K m_{e} c^{2} E\right)^{1 / 2}} .
$$

The total produced luminosity is proportional to $\int E^{-1 / 2} d E$ and peaks at the high-energy end as $E^{1 / 2}$. The most energetic escaping photons are emitted at $b \approx b_{0}$, near the boundary of the radiative zone, with energies comparable to $1 \mathrm{MeV}$. Equation (26) does not include the additional component that is generated near $1 \mathrm{MeV}$ by photon splitting; it will be included in the detailed Monte-Carlo models presented below.

In Section 2.3, the cascade without collective effects gave a softer spectrum $d L / d E \propto E^{-0.9}$ (Equation [20). In that case, $\bar{\gamma}(b)$ was not proportional to $\gamma_{\mathrm{sc}}$; the secondary particles with $\gamma<\gamma_{\text {sc }}$ kept constant $\gamma$ until they 
reached the scattering curve at lower $b$ and radiated their energy in softer photons. By contrast, the collective behavior with $\bar{\gamma} \propto \gamma_{\mathrm{sc}}$ invokes a quick redistribution of energy between the injected particles with $\gamma<\gamma_{\text {sc }}$ and the radiating particles with $\gamma \approx \gamma_{\mathrm{sc}}$.

\subsection{Detailed calculation: angular distribution}

To evaluate the emission received from an active magnetosphere by observers with various lines of sight, we need a more detailed calculation. A concrete geometry of the j-bundle must be chosen in such simulations.

One can view the active part of the magnetosphere (j-bundle) as a collection of infinitesimally thin active magnetic flux tubes. The flux tubes are assumed to extend sufficiently far from the neutron star, reaching the region of $B<10^{12} \mathrm{G}$. Consider one elementary tube that contains magnetic flux $\delta \digamma$ and electric current $\delta I$. The discharge operates in the tube under voltage $\Phi$ and creates an outflow with power,

$$
\delta L=\chi \Phi \delta I .
$$

Here we allowed a fraction $1-\chi$ of the total ohmic power released in the discharge zone to go to the footpoints of the tube, and the fraction $\chi$ is given to the outflowing particles.

Emission from each piece of the tube is beamed along the outflow, and the emerging radiation is strongly anisotropic. Consider a short piece of the magnetic tube of length $d l$ which corresponds to $d b=(d b / d l) d l$. Its contribution to the tube luminosity is $\left(\delta L / 2 b_{0}\right)|d b|$ (see Equation 24 and take into account that there are two elements $d l$ that correspond to $d b$, as the tube has two footpoints). The average energy of emitted photons is $\gamma_{\mathrm{sc}} b m_{e} c^{2}$, and hence the photon emission rate for the tube element $d l$ is given by

$$
d\left(\delta \dot{N}_{\mathrm{ph}}\right)=\frac{\delta L}{2 \gamma_{\mathrm{sc}} b m_{e} c^{2}} \frac{|d b|}{b_{0}} .
$$

It remains to determine the spectrum and polarization of the emitted photons. In the radiative zone $b$ is small $(b \ll 1)$ and hence electron recoil per scattering is small. In this regime, the description of polarization and angular distribution of scattered photons is simple (see Appendix A in B12 for details). Photon energy in the observer frame, $E$, is related to its emission angle in the electron rest frame, $\tilde{\vartheta}$, by the Doppler transformation of $\tilde{E}=\hbar \omega_{B}$,

$$
E=\hbar \omega_{B} \gamma_{\mathrm{sc}}\left(1+\beta_{\mathrm{sc}} \cos \tilde{\vartheta}\right)
$$

Approximately $3 / 4$ of scattered photons have the $\perp$ polarization (cf. Equation A3); these photons are emitted with an isotropic distribution,

$$
P_{\perp}(\cos \tilde{\vartheta})=\text { const }=\frac{1}{2} .
$$

The remaining $1 / 4$ of photons are emitted with the $\|$ polarization, and their angular distribution is

$$
P_{\|}(\cos \tilde{\vartheta})=\frac{3}{2} \cos ^{2} \tilde{\vartheta}
$$

Using the Monte-Carlo technique, we generate the angular distributions for photons with $\perp$ and $\|$ polarizations, and determine the photon energies according to Equation (29).

This straightforward Monte-Carlo simulation gives the spectrum and angular distribution of emission from all parts of the magnetic tube. We determine the fate of emitted photons by tracking their trajectories through the magnetosphere and checking for splitting or conversion to $e^{ \pm}$, which allows us to smoothly describe the transition from pair-loading to radiative zone (Appendix B). Photons that avoid splitting and conversion form the observed spectrum. Finally, the net observed emission is obtained by integrating the emission along the tube and then taking the sum of emissions from all tubes in the j-bundle.

The same calculation may be performed by integrating emission over the volume of the j-bundle in usual spherical coordinates. This method is described in Appendix $\mathrm{C}$, where emissivity per unit volume $d L / d V$ is derived. Appendix $\mathrm{C}$ also describes the concrete case of a twisted dipole magnetosphere.

\subsection{Multiple scattering of hard $X$-rays}

The Monte-Carlo simulation described above neglects the fact that the generated high-energy photons can scatter multiple times in the outflow before escaping. It turns out that this additional scattering weakly affects the emerging spectrum. Note two facts: (1) Hard X-rays escape the scattering region on the light-crossing time regardless of the number of scatterings; this is because the plasma moves relativistically and the scattered photons are beamed along the flow, so they keep moving away from the star. (2) The outflow significantly loses energy only when it scatters soft target photons that propagate at large angles $\vartheta$ with respect to the outflow direction; then scattering boosts the photon energy by the large factor of $\sim \gamma_{\mathrm{sc}}^{2} \gg 13$ Since the scattering reduces $\vartheta$ to $\sim \gamma_{\mathrm{sc}}^{-1}$ (which corresponds to a quasi-isotropic distribution of photons in the outflow rest frame), subsequent scatterings have a small effect on the photon energy.

To check the effect of multiple scattering on the hard X-ray spectrum we used the Monte-Carlo transfer code developed in the accompanying paper (B12). We followed the propagation and scattering of the generated hard X-rays through the flowing magnetospheric plasma and found that multiple scattering changes the emerging spectrum by less than $20 \%$. This change makes the spectrum slightly harder; it will be neglected in this paper.

\section{SAMPLE NUMERICAL MODELS}

\subsection{Model setup}

Consider a neutron star of radius $R=10 \mathrm{~km}$, temperature $k T=0.5 \mathrm{keV}$, and magnetic dipole moment $\hat{\mu}=5 \times 10^{32} \mathrm{G}$ (which corresponds to the magnetic field at the pole $B_{\text {pole }}=10^{15} \mathrm{G}$ ). Poloidal magnetic field is well approximated as dipole if the twist amplitude is moderate, $\psi \lesssim 1$ (B09; Parfrey et al. 2012b). We will use spherical coordinates $r, \theta, \phi$ with the polar axis aligned with the dipole moment; $\theta=0$ on the axis.

Consider an activated magnetic loop (j-bundle) whose

3 This boost happens in the lab frame; the photon energy is unchanged in the outflow rest frame - only its angle is changed by scattering (from $\cos \tilde{\vartheta} \approx-1$ to a random value). 
northern footpoint on the star is defined by

$$
\frac{\theta_{\star}}{2}<\theta<\theta_{\star}, \quad 0<\phi<\phi_{\star} .
$$

In our sample models, $\sin ^{2} \theta_{\star}=0.1 ; \phi_{\star}$ is left as a free parameter $\left(\phi_{\star}=2 \pi\right.$ describes an axisymmetric j-bundle). Practically the same results are obtained if the j-bundle includes the entire polar cap $0<\theta<\theta_{\star}$, as the field lines with footpoints at $\theta<\theta_{\star} / 2$ contribute only a small fraction $\sim 2^{-4}$ to the emitted luminosity 4 The active loop extends to radii $r \gtrsim R / \sin ^{2} \theta_{\star} \approx 10 R$. We wish to know the spectrum of radiation emitted by the loop, as viewed along any given line of sight.

The total luminosity of the loop is controlled by its twist amplitude $\psi$ and the discharge voltage $\Phi$ (Section 2.1). It is approximately given by (see B09 and Appendix C)

$$
L \approx \frac{c \Phi \hat{\mu} \psi \sin ^{4} \theta_{\star}}{4 R^{2}}\left(\frac{\phi_{\star}}{2 \pi}\right) .
$$

The discharge occurs in both hemispheres, forming two outflows that meet and annihilate at the equatorial plane of the magnetic dipole. Therefore, the total emitted power corresponds to $\Phi$ that is two times larger than the threshold for the discharge; $\Phi=10^{9}-10^{10} \mathrm{~V}$ is expected (Beloborodov \& Thompson 2007) and consistent with observations (Beloborodov 2011). It is convenient to define the parameter

$$
H \equiv \psi\left(\frac{\Phi}{3 \times 10^{9} \mathrm{~V}}\right)\left(\frac{\phi_{\star}}{2 \pi}\right)\left(\frac{R}{10 \mathrm{~km}}\right) .
$$

Then the total power emitted by the loop may be written as

$$
L \approx 4 \times 10^{35} H\left(\frac{B_{\text {pole }}}{10^{15} \mathrm{G}}\right)\left(\frac{\sin ^{4} \theta_{\star}}{10^{-2}}\right) \mathrm{erg} \mathrm{s}^{-1} .
$$

The observed emission from the loop is calculated using the Monte-Carlo technique as described in Section 3.4. We assume that the $e^{ \pm}$outflow behaves collectively (Section 3.3), as this model is both simpler and more realistic than the cascade without collective effects.

The emission from the loop is strongly anisotropic and hence emission observed along a given line of sight will be modulated by the rotation of the object. Let us choose the fixed (non-rotating) lab frame so that its $z$-axis is parallel to the spin axis, and the $x z$ plane contains the observer's line of sight. The instantaneous orientation of the magnetosphere with respect to the lab frame is described by three Euler angles $\alpha, \varphi, \zeta$. Here $\alpha$ is the angle between the magnetic and rotation axes, $\varphi$ is the angle of rotation about the spin axis, and $-\zeta=\phi_{\text {nodes }}$ is the azimuthal angle (measured in magnetic coordinates $r, \theta, \phi)$ of the line of nodes $\vec{\Omega} \times \overrightarrow{\hat{\mu}}$.

Let $\beta$ be the angle between the line of sight and the rotation axis. The instantaneous observed emission is determined by four angles $\alpha, \varphi, \zeta$, and $\beta$, and the observed spectral luminosity $L_{E}$ varies periodically with $\varphi$. The magnitude of the variation may be described by the

\footnotetext{
4 Electric current in the j-bundle $\theta<\theta_{\star}$ with a given twist $\psi$ scales as $I \propto \psi \sin ^{4} \theta_{\star}$ (Appendix C).
}

"pulsed fraction,"

$$
f_{p}(E) \equiv \frac{L_{E}^{\max }-L_{E}^{\min }}{L_{E}^{\max }+L_{E}^{\min }},
$$

or the "pulsed spectrum,"

$$
L_{E}^{\mathrm{puls}} \equiv L_{E}^{\max }-L_{E}^{\min } .
$$

If the emission is not resolved in rotational phase, its observed spectrum is obtained by averaging the instantaneous $L_{E}$ over the rotation period,

$$
\bar{L}_{E}=\frac{1}{2 \pi} \int_{0}^{2 \pi} L_{E} d \varphi .
$$

The result depends on $\alpha, \beta$, and $\zeta$. If the emitting region (j-bundle) is symmetric about the magnetic axis, the result only depends on $\alpha$ and $\beta$.

\subsection{Aligned rotator}

Aligned rotator has $\alpha=0$ (magnetic axis parallel to the rotation axis). Then $\varphi=\phi$ and averaging over rotation is the same as averaging over the magnetic azimuthal angle $\phi$. The shape of the averaged spectrum $\bar{L}_{E}$ does not depend on the azimuthal extension of the j-bundle $\phi_{\star}$; e.g. one may chose $\phi_{\star}=2 \pi$ (axisymmetric j-bundle).

The spectrum produced by the axisymmetric $\mathrm{j}$-bundle is shown in Figure 7. It strongly depends on the angle between the line of sight and the magnetic axis. In particular, the position of the high-energy peak significantly varies around $1 \mathrm{MeV}$. One can qualitatively understand the variations in the spectrum with changing inclination by combining the following facts. (1) Emission from the loop is generated on field lines that have footpoints at colatitudes $\theta \sim 0.3$ and reach the equatorial plane at radii $R_{\max }=10-20$. (2) Emission is strongly beamed outward along the magnetic field lines. (3) Photons of a given energy $E$ are mainly generated where $b \approx 0.1\left(E / m_{e} c^{2}\right)^{1 / 2}$ (see Equation 25). Note also that observers in the equatorial plane (inclination $\theta=90^{\circ}$ ) are special - by symmetry, both hemispheres equally contribute to the observed spectrum. At small inclinations, one hemisphere strongly dominates hard X-ray emission; however, the contribution of the other hemisphere is still significant in the soft-X-ray band.

If the emission shown in Figure 7 is averaged over cosine of inclination angle one obtains the spectrum shown in Figure 8. Below $1 \mathrm{MeV}$, it approximately follows the power-law $E L_{E} \propto E^{1 / 2}$ in agreement with Equation (26) derived in Section 3.4. It corresponds to photon index $\Gamma=1.5$ (which is defined by $L_{E} \propto E^{-\Gamma+1}$ ). This does not imply that, given a random inclination, the most probable observed spectrum has $\Gamma=1.5$. Significantly harder slopes $\Gamma \sim 1$ are observed in Figure 7 . In a range of inclinations, the spectra have breaks well below $1 \mathrm{MeV}$, and after averaging over inclination this gives the featureless power law with a softer slope $\Gamma=1.5$.

Approximately $3 / 4$ of photons scattered in the j-bundle have the $\perp$ polarization. Therefore at energies $E<$ $1 \mathrm{MeV}$ (where the splitting of $\perp$ photons is negligible), the produced emission is dominated by the $\perp$ polarization, see the lower panel in Figure 8.

The inclination-averaged spectrum exhibits a significant hump at $E \gtrsim 1 \mathrm{MeV}$. It is the result of photon split- 


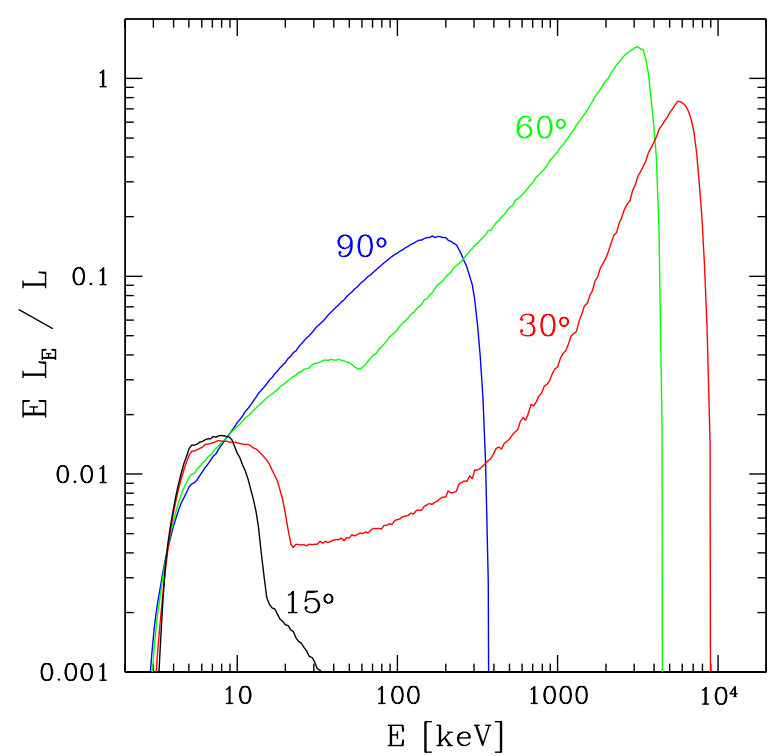

Figure 7. Spectrum from the axisymmetric j-bundle, observed at four different angles with respect to the magnetic axis: $15^{\circ}, 30^{\circ}$, $60^{\circ}$, and $90^{\circ}$. The spectrum is normalized to the total luminosity of the j-bundle $L$ given by Equation (35).
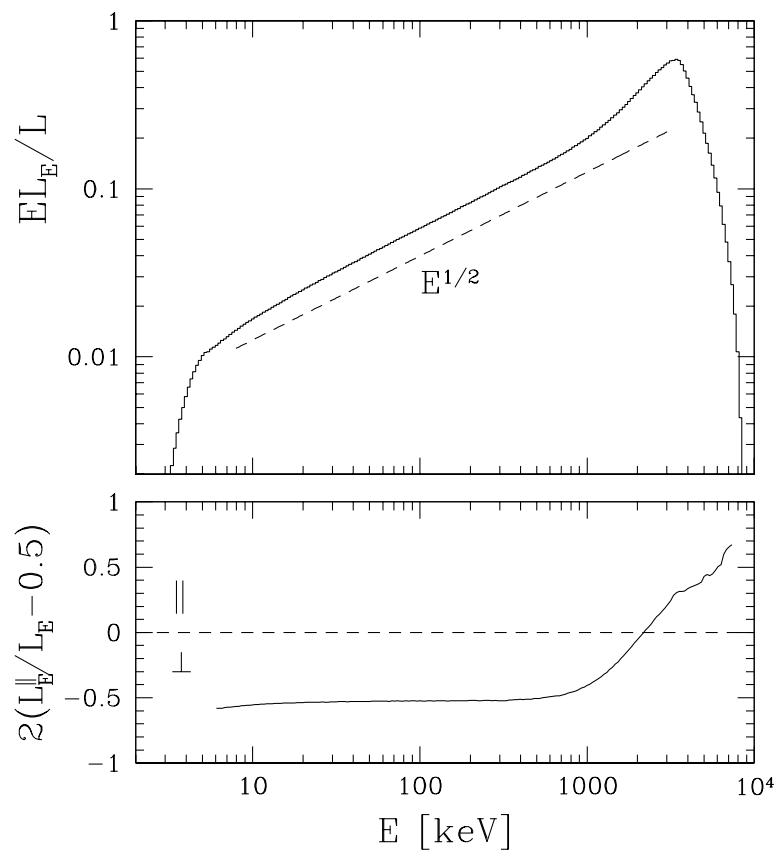

Figure 8. Upper panel: spectrum from the axisymmetric jbundle, $L_{E}$, averaged over the object inclination to the line of sight. Lower panel: relative contributions of $\perp$ and $\|$ to the total spectrum $L_{E}=L_{E}^{\perp}+L_{E}^{\|}$.

ting. The daughter photons produced by splitting have the $\|$ polarization, and the splitting-dominated component is particularly visible in the lower panel of Figure 8 that shows the polarization of emitted photons. Note that Figure 8 does not take into account the possible multiple scattering of high-energy photons on their way out of the j-bundle (Section 3.5). Additional scattering weakly changes the spectrum of escaping radiation, how- ever it can significantly affect polarization of the $\mathrm{MeV}$ hump. Scattering of daughter photons after splitting switches polarization from $\|$ to $\perp$ with probability of $3 / 4$ and tends to bring the polarization to the same value as below $1 \mathrm{MeV}$.

No pulsations are produced by the aligned rotator if the $\mathrm{j}$-bundle is axially symmetric, $\phi_{\star}=2 \pi$. Strong pulsations are produced if $\phi_{\star}<2 \pi$, i.e. if the $\mathrm{j}$-bundle is confined to a smaller range of azimuthal angles. The pulsations are the consequence of strong beaming of highenergy emission along the magnetic field lines, which makes the emission almost invisible when the line of sight is outside the interval $0<\phi<\phi_{\star}$. The pulse width is proportional to $\phi_{\star}$, and the pulse fraction can approach $100 \%$.

\subsection{Orthogonal rotator}

Orthogonal rotator has $\alpha=\pi / 2$ (magnetic axis is perpendicular to the rotation axis). Then even an axisymmetric j-bundle $\left(\phi_{\star}=2 \pi\right)$ will produce pulsating emission. As the magnetar rotates, the inclination of line of sight to the magnetic axis, $\theta$, periodically changes and the observer samples the spectra shown in Figure 7 . The spectrum strongly depends on $\theta$ and hence significant periodic variations in $L_{E}$ are expected, depending on $\beta$ (the angle between the line of sight and the rotation axis). In particular, the position of the high-energy peak will oscillate with a significant amplitude. No pulsations are expected only if $\beta=0$; the observed spectrum in this case is the same as the $\theta=\pi / 2$ spectrum in Figure 7 .

Figure 9 shows the emission from the orthogonal rotator viewed at $\beta=20^{\circ}$. The rotation-averaged spectrum has the photon index $\Gamma \approx 1$ at photon energies below $70 \mathrm{keV}$. The pulsed spectrum is significantly harder, $\Gamma \approx 0$. The pulsed fraction increases with $E$ and approaches $100 \%$ at $E \approx 300 \mathrm{keV}$. The model is close to observational data reported for $4 \mathrm{U} 0142+61$ and 1RXS J170849-400910 - two magnetars whose hard X-ray pulsations have been studied in detail (den Hartog et al. 2008a,b).

Figure 10 shows the emission from the orthogonal rotator viewed at $\beta=90^{\circ}$. The rotation-averaged spectrum has the photon index $\Gamma \approx 1.5$ below $1 \mathrm{MeV}$; this spectrum is close to the average spectrum in Figure 8, although not exactly the same 5 The pulsed spectrum is again harder than the average spectrum. The amplitude of pulsations is significantly larger than in Figure 9; the pulsed fraction approaches $100 \%$ at $E \approx 20 \mathrm{keV}$.

The variety of observed spectra and pulse profiles becomes even larger if the $\mathrm{j}$-bundle is not axisymmetric. Then the observed emission depends on the third Euler angle $\zeta$ (Section 4.1). The pulse profile in a given spectral window depends on the angles $\alpha, \beta, \zeta$ and the geometry of the j-bundle.

\section{DISCUSSION}

In this paper, we calculated the X-ray emission expected from large twisted magnetic loops around magnetars. The loops are inevitably heavily loaded with $e^{ \pm}$

5 For the orthogonal rotator viewed at $\beta=90^{\circ}$, we have $\theta=\varphi$, where $\theta$ is the angle between the line of sight and the magnetic axis. Therefore, averaging over rotation $0<\varphi<2 \pi$ is equivalent to averaging over $\theta$. In Figure 8, the emitted spectrum was averaged over $\cos \theta$ (rather than $\theta$ ), giving a slightly different result. 


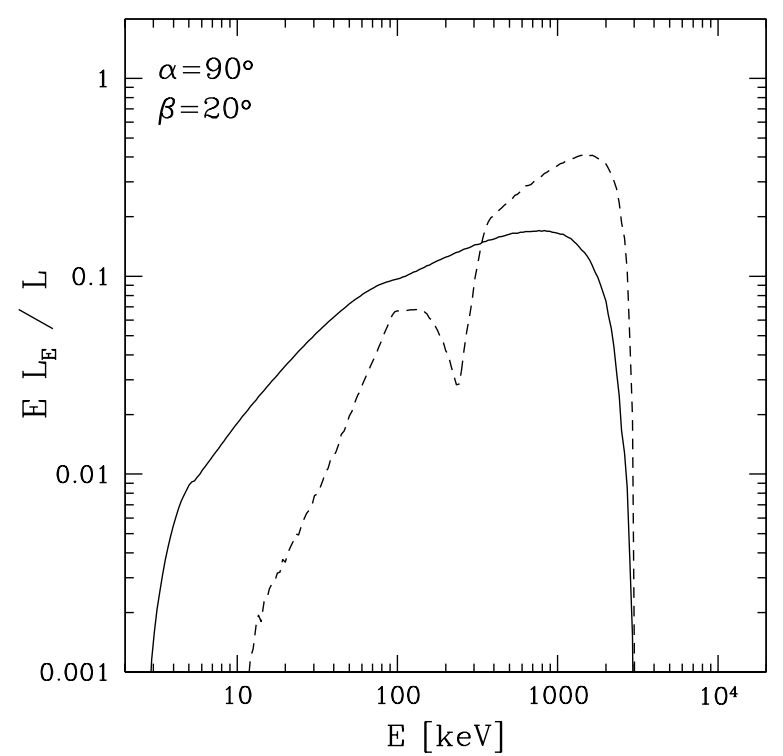

Figure 9. Spectrum from the orthogonal rotator $\left(\alpha=90^{\circ}\right)$ when the line of sight is at angle $\beta=20^{\circ}$ with respect to the spin axis. The $\mathrm{j}$-bundle is assumed to be symmetric about the magnetic axis $\left(\phi_{\star}=2 \pi\right)$. Solid curve shows the spectrum averaged over rotation (Equation 38). Dashed curve shows the pulsed spectrum (Equation 37.

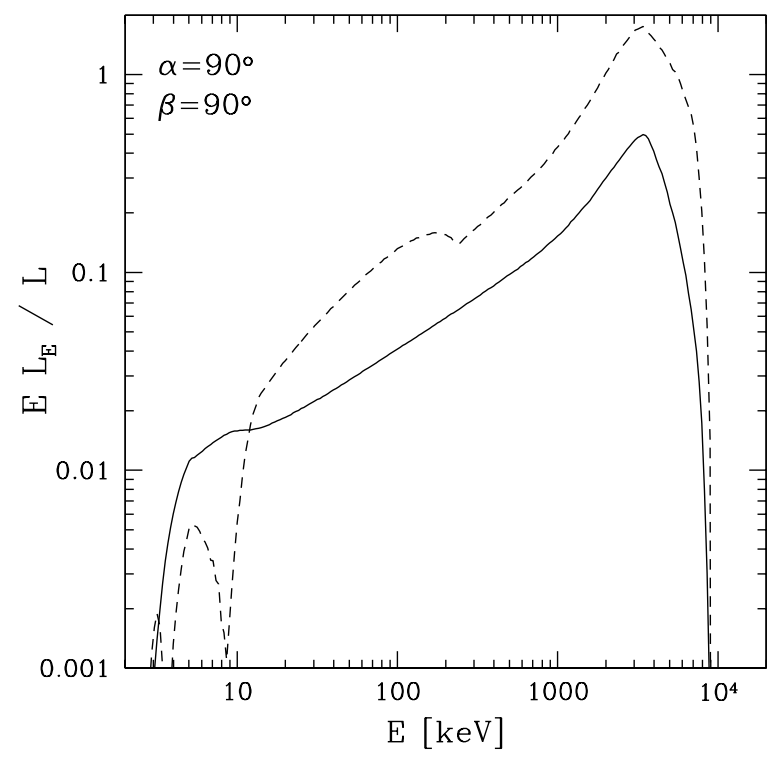

Figure 10. Same as Figure 9 but for $\beta=90^{\circ}$.

pairs, with multiplicity $\mathcal{M} \sim 10^{2}$, which leads to efficient screening of electric fields. The created plasma outflows along the magnetic loop and loses its energy to radiation. Transformation of the outflow energy to escaping radiation occurs in the region $10^{13}>B>3 \times 10^{11} \mathrm{G}$, which requires a minimum size of the loop 3 to 10 stellar radii (if the surface field is $\sim 10^{15} \mathrm{G}$ ). We did not consider small loops because most of photons scattered near the star are unable to escape the magnetosphere; besides, smaller loops are less likely to be active (Section 2.1).

A key feature of the $e^{ \pm}$outflow along the loop is that its Lorentz factor is self-regulated so that it scatters ra- diation with $\gamma_{\mathrm{sc}} \approx 100 B / B_{Q}$ (Section 2.2$)$. This fact determines the radiation spectrum emitted by the outflow, taking into account that the scattered photons have the characteristic energy $E \sim \gamma_{\mathrm{sc}}\left(B / B_{Q}\right) m_{e} c^{2}$. The emitted spectrum below $1 \mathrm{MeV}$ is hard, i.e. softer photons $E \ll 1 \mathrm{MeV}$ contribute less to the outflow luminosity. This is simply because softer photons are emitted where the outflow decelerates, and there remains less energy to emit. The spectrum is also suppressed at energies $E \gg 1 \mathrm{MeV}$, because photons of such high energies are emitted in the zone of strong $B$ and cannot escape.

The model predictions appear to agree with available data. Observations of magnetars in the 10-100 keV band show a typical photon index $\Gamma \sim 1-1.5$, similar to what is found in the model spectra. The observed spectra peak above $100 \mathrm{keV}$, and the existing upper limits for AXP 4 U $0142+61$ at 1-30 MeV indicate a spectral break between 0.3 and $1 \mathrm{MeV}$ (den Hartog et al. 2008a).

The strong beaming of radiation along the loop explains why the observed hard X-ray flux shows huge pulsations, with the pulsed fraction growing with photon energy up to $100 \%$ (Kuiper et al. 2003; den Hartog et al. 2008a,b). No unique pulse profile is predicted by our model, because the pulse is sensitive to the loop geometry, its orientation with respect to the rotation axis and the line of sight. The pulse profile may be further complicated if more than one loop are activated and the superposition of their emissions is observed. The expected hard X-ray pulse does not have to be in phase with the soft X-ray pulse, as observed in AXP 1RXS J170849400910 (see Figure 6 in Den Hartog et al. 2008b).

In general, sources of beamed, pulsed radiation are not expected to display a standard spectrum at all viewing angles, and magnetars are not an exception. The active loop typically emits a hard spectrum, $\Gamma \sim 1-3 / 2$, but its detailed shape depends on the loop and the viewing angle (Figures 7, 9, 10). The predicted high-energy peak significantly depends on the line of sight and its observed position is expected to oscillate as the magnetar rotates.

Note also that a very hard spectrum $\Gamma<1$ may be produced if smaller magnetic loops are activated. For example, consider a loop that is confined to the region of strong magnetic field $B>3 \times 10^{12} \mathrm{G}$, where $\hbar \omega_{B}>$ $35 \mathrm{keV}$. The loop generates photons of energy $E \sim \gamma \hbar \omega_{B}$ and almost no emission is produced below $35 \mathrm{keV}$, leading to a hard spectral slope.

The surface transition layer between the corona and the star may give a second contribution to the hard X-ray emission (Thompson \& Beloborodov 2005; Beloborodov \& Thompson 2007). This dense layer is bombarded by the particles from the discharge and may reach temperatures $k T \gtrsim 100 \mathrm{keV}$, sufficient to emit high-energy bremsstrahlung radiation with photon in$\operatorname{dex} \Gamma \approx 1$. Observed magnetars show complex spectral variations with rotational phase, which suggests that the emitting plasma occupies an extended region in the magnetosphere rather than a heated spot on the surface. This favors the outflow model described in the present paper. Polarization measurements would provide a further test discriminating between the two mechanisms. The surface transition layer emits photons with the II polarization, while the loop itself emits radiation that is dominated by the $\perp$ polarization (also called "extraordinary" or E-mode). Careful calculations of polarization 
should include transfer effects (Fernández \& Davis 2011).

\subsection{Annihilation line}

The outflowing $e^{ \pm}$pairs accumulate and annihilate at the top of the magnetic loop with Lorentz factors $\gamma \sim 1$ (Figure 1). This must create an annihilation line at $E \approx 0.511 \mathrm{MeV}$, which we did not show in our sample spectra. There is a simple relation between the luminosity of annihilation radiation $L_{\text {ann }}$ and the total outflow power $L$ (which is equal to the luminosity of the hard X-ray component). The outflowing particles enter the radiative zone $B<10^{13} \mathrm{G}$ with the average Lorentz factor $\bar{\gamma}_{0} \sim 10-20$ and radiate almost all their kinetic energy $\left(\bar{\gamma}_{0}-1\right) m_{e} c^{2}$ in hard X-rays before annihilating. Therefore, a fraction of $\sim \bar{\gamma}_{0}^{-1}$ of the total outflow energy is converted to annihilation radiation,

$$
\frac{L_{\mathrm{ann}}}{L}=\frac{1}{\bar{\gamma}_{0}} \sim 0.1
$$

The visibility of the annihilation line at $511 \mathrm{keV}$ depends on its width. As $e^{ \pm}$are decelerated before annihilating, a pronounced narrow line is expected. Mildly relativistic motions in the annihilation region would make the line broad and less visible. Note also that $L_{\text {ann }}$ is approximately isotropic, while $L$ is strongly anisotropic; therefore the observed ratio $L_{\text {ann }} / L$ will depend on the line of sight and can be significantly larger or smaller than the mean expectation given by Equation (39).

\subsection{Temporal behavior of activity}

The nonthermal emission of magnetars must be fed by ohmic dissipation of electric currents excited in their twisted magnetospheres (Section 2.1). The rate of energy release is proportional to voltage established along the magnetic field lines, which is regulated by electric discharge to $\Phi \sim 10^{9} \mathrm{~V}$, in agreement with the observed luminosity. One expects the untwisting magnetosphere and its luminosity to evolve on the ohmic timescale, which is comparable to 1 year. Several transient magnetars show evidence for this evolution (Beloborodov 2011); XTE J1810-197 is a canonical example. Hard X-ray data for transient magnetars are meager. A strong evolution of the hard X-ray flux is expected in these objects, which may be tested by the upcoming $N u S T A R$ observations.

Other sources, in particular 4U $0142+61$ and 1RXS J170849-400910, remain stable over a long observation period (about 10 years). Their hard X-ray emission has been studied in detail (den Hartog et al. 2008a,b). The different patterns of magnetar activity are not understood because of our ignorance concerning the pattern and speed of the crustal motions that cause the activity. The crust is likely to flow plastically and gradually twist the magnetosphere. It is unclear whether a quasisteady state should be expected, punctuated by sudden outbursts triggered by excessive twisting (Parfrey et al. 2012b).

\subsection{1-10 ke V emission}

In this paper, we focused on the mechanism for the hard X-ray peak in magnetar spectra, and did not address the shape of the soft X-ray (keV) peak. As has been known for a long time, the $\mathrm{keV}$ peak is different from simple blackbody (e.g. Woods \& Thompson 2006). The $1-10 \mathrm{keV}$ spectrum is explained as a superposition of the thermal component and a soft power-law tail with photon index $\Gamma=2-4$. The soft tail is distinct from the hard X-ray component, although a correlation between them was reported (Kaspi \& Boydstun 2010).

Previous models of resonant scattering around magnetars were able to reproduce the soft tail in the 1$10 \mathrm{keV}$ band (Fernández \& Thompson 2007; Nobili, Turolla, \& Zane 2008; Rea et al. 2008). In these models, the magnetospheric region where $\hbar \omega_{B} \sim 1-10 \mathrm{keV}$ $\left(B=10^{11}-10^{12} \mathrm{G}\right)$ is filled with a mildly relativistic plasma $(\gamma \beta \sim 1)$. It is usually assumed that the plasma is made of counter-streaming positive and negative charges, which maintain the electric current with particle multiplicity $\mathcal{M} \sim 1$; then the scattering optical depth can be estimated as $\tau \sim \psi / \beta \sim 1$ (Thompson et al. 2002 ). The results of the present paper show that these assumptions are problematic. The typical pair multiplicity $\mathcal{M}$ must be comparable to $10^{2}$, and charges of both signs should stream away from the star, toward their annihilation at the top of the magnetic loop. The model of the soft X-ray tail due to resonant scattering should be revised accordingly.

The high multiplicity alleviates the following problem. The particle flux along the magnetic field lines is $\dot{N}=$ $\mathcal{M} I /$ e where $I / e \sim 5 \times 10^{37} \psi \mathrm{s}^{-1}$ is a typical electric current circulating through the region $\hbar \omega_{B} \sim 3 \mathrm{keV}$ (cf. Equation (1). The observed luminosity in the soft tail $L_{\text {tail }}$ is comparable to $10^{35} \mathrm{erg} \mathrm{s}^{-1}$, and hence the energy emitted per particle is given by

$$
\frac{L_{\text {tail }}}{2 \dot{N}}=\frac{e L_{\text {tail }}}{2 I \mathcal{M}} \sim 600 \mathcal{M}^{-1}\left(\frac{L_{\text {tail }}}{10^{35} \mathrm{erg} \mathrm{s}^{-1}}\right) \mathrm{MeV}
$$

The mildly relativistic particles that are supposed to produce $L_{\text {tail }}$ have energies $\sim 1 \mathrm{MeV}$. Hence the picture of counter-streaming charges with $\mathcal{M} \sim 1$ requires that the emitted energy is 2-3 orders of magnitude larger than the particle energy 6 In contrast, the model with multiplicity $\mathcal{M} \gtrsim 10^{2}$ implies that a few $\mathrm{MeV}$ per particle is sufficient to feed the observed $L_{\text {tail }}$ and modify the thermal peak.

This work was supported by NASA NNX10AI72G.

\footnotetext{
${ }^{6}$ One could assume that a voltage $e \Phi \sim 1 \mathrm{GeV}$ is applied to this region and responsible for the enhanced emission rate per particle. In this picture, radiative drag impedes the electrostatic acceleration and keeps the particles mildly relativistic. This "radiatively locked
}

flow" is considered in more detail in the accompanying paper (B12). Note also that the balance between the radiative and electrostatic forces is unstable when the particle interacts with photons below the Wien peak of thermal radiation. 


\section{APPENDIX}

\section{RESONANT SCATTERING}

\section{Cross section, photon energy, and polarization}

The polarization states of X-rays in the magnetosphere are determined by the quantum electrodynamic effect of vacuum polarization in a strong magnetic field. It defines two eigen modes for an electromagnetic wave with a wavevector $\mathbf{k}$ : with electric field oscillating along $\mathbf{B} \times \mathbf{k}(\perp$ polarization, sometimes called "extraordinary" or E-mode) and with electric field oscillating along $\mathbf{k} \times(\mathbf{B} \times \mathbf{k})$ (\| polarization, sometimes called "ordinary" or O-mode).

In our problem, the outflow is ultra-relativistic in the region of strong $B$, and target photons are strongly aberrated to become nearly parallel to $\mathbf{B}$ in the electron rest frame. Then the scattering cross section is the same for $\|$ and $\perp$ photons. Let $\tilde{E}_{t}=\hbar \tilde{\omega}$ be the energy of a target photon measured in the electron rest frame. Resonant scattering cross section in this frame is given by

$$
\tilde{\sigma}=2 \pi^{2} r_{e} c \delta\left(\tilde{\omega}-\omega_{B}\right), \quad \omega_{B}=\frac{e B}{m_{e} c},
$$

where $r_{e}=e^{2} / m_{e} c^{2}$. Equation A1) can be derived classically when $B \ll B_{Q}$ (e.g. Canuto et al. 1971; Ventura 1979). It also remains valid at any $B$, as long as target photons propagate nearly along $\mathbf{B}$ in the electron rest frame (e.g. Harding \& Daugherty 1991).

Consider an electron moving along a magnetic field line with Lorentz factor $\gamma=\left(1-\beta^{2}\right)^{-1 / 2}$. When the electron scatters a photon, the photon energy changes from $E_{t}$ to $E$. Energy conservation gives $\left(\gamma-\gamma_{f}\right) m_{e} c^{2}=E-E_{t}$ where $\gamma_{f}$ is the electron Lorentz factor after scattering. Resonant scattering may be viewed as photon absorption, which excites the electron to the first Landau level $E_{B}=(1+2 b)^{1 / 2} m_{e} c^{2}$, followed by photon emission (electron de-excitation). The Lorentz factor of the excited electron $\gamma_{1}$ is found from $\gamma m_{e} c^{2}+E_{t}=\gamma_{1} E_{B}$. Assuming that de-excitation on average does not decelerate or accelerate the electron along $\mathbf{B}$, the mean expectation for $\gamma_{f}$ is $\gamma_{1}$. Then the average energy of emitted photons is $E=\left(\gamma m_{e} c^{2}+E_{t}\right)\left(1-m_{e} c^{2} / E_{B}\right)$, which yields (using $\left.E_{t} \ll \gamma m_{e} c^{2}\right)$,

$$
E \approx \gamma m_{e} c^{2}\left(1-\frac{1}{\sqrt{1+2 b}}\right), \quad b=\frac{B}{B_{Q}} .
$$

This expression gives a typical energy of scattered photons; the exact $E$ in each scattering event depends on the scattering angle.

The scattered photons can be in one of the two polarization states. Using the accurate relativistic differential cross section, we find that the fraction of scattered photons with the $\perp$ polarization is conveniently approximated by the following formula,

$$
\frac{\sigma_{\perp}}{\sigma_{\perp}+\sigma_{\|}} \approx \frac{3+b / 2}{4+b}
$$

\section{Scattering rate}

Let $n_{\omega}(\vec{\Omega}, \omega)$ be the local angular distribution of the photon spectral density $\left[\mathrm{cm}^{-3} \mathrm{~s}\right]$. The cross section (A1) implies the following rate of resonant scattering by one electron,

$$
\dot{N}_{\mathrm{sc}}=\frac{2 \pi^{2} r_{e} c^{2}}{\gamma} \int d \Omega \int d \omega n_{\omega} \delta\left(\omega-\omega_{\mathrm{res}}\right)
$$

where $\omega_{\text {res }}=\gamma(1-\vec{\beta} \cdot \vec{\Omega}) \omega_{B}$. In our problem, the spectrum of target radiation seen by the electron may be approximated by a (diluted) Planckian with temperature $T$ and photon density $n$,

$$
\omega n_{\omega} \approx 0.42 \frac{y^{3}}{e^{y}-1} n f(\vec{\Omega}), \quad y=\frac{\hbar \omega}{k T} .
$$

Then $\dot{N}_{\text {sc }}$ becomes,

$$
\dot{N}_{\mathrm{sc}} \approx \frac{2 \pi^{2} r_{e} c^{2} \hbar}{\gamma k T} 0.42 n \int f(\vec{\Omega}) \frac{y_{\mathrm{res}}^{2}}{\exp y_{\mathrm{res}}-1} d \Omega .
$$

The resonance condition defines the range of possible $y_{\text {res }}, y_{\min }<y_{\text {res }}<y_{\max }$. When $y_{\min } \gg 1$, only photons in the Wien tail of the Planck spectrum can be scattered. Then the integral in Equation (A6) strongly peaks where $y_{\text {res }} \approx y_{\text {min }}$, which gives (using $f[\vec{\Omega}] d \Omega \sim y_{\text {res }}^{-1} d y_{\text {res }}$ ),

$$
\dot{N}_{\mathrm{sc}} \approx \frac{2 \pi^{2} r_{e} c^{2} \hbar}{\gamma k T} 0.42 n y_{\min } e^{-y_{\min }}, \quad y_{\min }=\frac{\hbar \omega_{B}}{\gamma\left(1-\beta \cos \vartheta_{\max }\right) k T},
$$

where $y_{\min }$ corresponds to the largest photon angle $\vartheta_{\max }$ with respect to $\vec{\beta}$. Thermal photons reflected by opaque plasma near the top of magnetic loops (Figure 1) have the maximum angle, as they stream against the outflow 
direction. The exponential sensitivity of $\dot{N}_{\mathrm{sc}}$ to $\vartheta_{\max }$ explains why the reflected photons are the main targets for scattering even though their total number density $n$ is much smaller than that of photons streaming directly from the star. For photons streaming at smaller angles $\vartheta, y_{\min }$ would increase, leading to an exponential suppression of $\dot{N}_{\text {sc }}$. For instance, changing $y_{\min }=7$ to $y_{\min }=14$ reduces $\dot{N}_{\mathrm{sc}}$ by the factor of $4 \exp (-7) \approx 4 \times 10^{-3}$.

The density of reflected thermal radiation that should be used in Equation (A7) is given by

$$
n \sim \frac{\xi L_{\mathrm{th}}}{4 \pi R_{1}^{2} 2.7 k T c} \approx 10^{17}\left(\frac{\xi}{0.1}\right)\left(\frac{L_{\mathrm{th}}}{10^{35} \mathrm{erg} \mathrm{s}^{-1}}\right)\left(\frac{k T}{0.5 \mathrm{keV}}\right)^{-1}\left(\frac{R_{1}}{100 \mathrm{~km}}\right)^{-2} \mathrm{~cm}^{-3} .
$$

Here $L_{\mathrm{th}}$ is the thermal luminosity of the star, $2.7 k T \sim 1 \mathrm{keV}$ stands for the average energy of thermal photons, and $\xi$ is the fraction of $L_{\text {th }}$ that is intercepted by the slow, opaque plasma at the top of magnetic loops.

In the calculations in this paper, it is convenient to describe the number of thermal photons scattered by an outflowing particle as a function of decreasing magnetic field,

$$
\frac{d N_{\mathrm{sc}}}{d B}=\frac{1}{\beta c} \frac{\dot{N}_{\mathrm{sc}}}{d B / d l},
$$

where $l$ is length measured along the magnetic field line, and $d B / d l$ (for dipole field lines) is given in Equation (C8); in the simulations shown in Figures 2 and 3 we use a simplified $d B / d l=-3 B / r$.

Drag exerted by the reflected radiation on a relativistic electron

The electron loses energy to radiation with rate

$$
\dot{E}_{e} \approx-\dot{N}_{\mathrm{sc}}\left(E-E_{t}\right)
$$

where $E$ is the average energy of scattered photons (Equation $\underline{A 2}$ ) and $E_{t}$ may be neglected compared with $E$. This gives

$$
\frac{\dot{E}_{e}}{m_{e} c^{3}} \approx-\frac{n \sigma_{\mathrm{T}}}{\alpha} \frac{m_{e} c^{2}}{k T} y_{\min } e^{-y_{\min }}\left(1-\frac{1}{\sqrt{1+2 b}}\right)
$$

where $\alpha=e^{2} / \hbar c=1 / 137$. The corresponding drag force acting on the relativistic electron is given by $\mathcal{F} \approx \dot{E}_{e} / c$.

The expression for $\dot{E}_{e}$ may be written in a different, physically more transparent form,

$$
\dot{E}_{e} \approx-c \sigma_{\mathrm{res}} n_{t} E
$$

Here $\sigma_{\text {res }}$ is the effective cross section for resonant scattering,

$$
\sigma_{\mathrm{res}} \approx(1-\beta \cos \vartheta) \pi r_{e} \tilde{\lambda}
$$

where $\tilde{\lambda}=2 \pi c / \omega_{B}$ is the photon wavelength in the rest frame of the electron. The factor $(1-\beta \cos \vartheta)$ (from transformation of cross section to the lab frame) is comparable to unity. $n_{t}$ in Equation (A12) is the density of photons near the resonant energy $\hbar \omega_{\text {res. }}$. Our electron interacts with photons in the Wien tail of the spectrum, and their number peaks near the minimum $y_{\text {res }}=\hbar \omega_{\text {res }} / k T$,

$$
\left.n_{t} \approx k T \frac{d n}{d E_{t}}\right|_{y_{\min }} \approx 0.42 y_{\min }^{2} e^{-y_{\min }} n .
$$

Substitution of Equations (A13) and (A14) to Equation (A12) gives an explicit expression for the drag force $\mathcal{F}=\dot{E}_{e} / c$. The estimate for $\mathcal{F}$ may be summarized in the following form,

$$
\frac{r \mathcal{F}}{\gamma m_{e} c^{2}} \approx \frac{3 \pi}{4} \frac{\sigma_{\mathrm{T}} n_{t} r}{\alpha} q\left(1-\beta \cos \vartheta_{\max }\right) \sim-10 \frac{r}{R} q n_{17} y_{\min }^{2} e^{-y_{\min }},
$$

where $n_{17}=n / 10^{17} \mathrm{~cm}^{-3}$. The recoil correction factor $q(b)=1$ when $b \ll 1$ (Equation 7 ). The approximate estimate given in Equation (A15) is sufficient in view of the strong exponential dependence on $y_{\text {min }}$ and the large prefactor $10(r / R) n_{17} y_{\min }^{2}$. The outflowing particles surf with $r \mathcal{F} / \gamma m_{e} c^{2} \sim|d \ln B / d \ln r| \sim 3$ (Section 2.2), which corresponds to $y_{\min } \sim 7$ and the prefactor $\sim 10^{3}$.

\section{PHOTON SPLITTING AND CONVERSION TO ELECTRON-POSITRON PAIRS \\ Conversion to $e^{ \pm}$}

Consider a photon of energy $E$ propagating at an angle $\vartheta$ with respect to the magnetic field lines. The photon can convert an $e^{ \pm}$pair if the threshold condition $E_{\mathrm{thr}}<E$ is satisfied. The threshold energy depends on the photon polarization: $E_{\mathrm{thr}}=2 m_{e} c^{2} / \sin \vartheta$ for $\|$ photons (then both $e^{+}$and $e^{-}$can be created in the ground Landau state) and $E_{\mathrm{thr}}=2 m_{e} c^{2}(1+2 b)^{1 / 2} / \sin \vartheta$ for $\perp$ photons (then at least one of the created particles is required to be in an excited Landau state). 
The rate of conversion depends on the local magnetic field $b=B / B_{Q}$. If $b \gtrsim 0.05$, one can assume practically instantaneous conversion once the thershold condition is satisfied; then the $e^{ \pm}$pair is created with the lowest possible energy $E_{-}+E_{+}=E_{\mathrm{thr}}$. If $b \lesssim 0.05$, conversion is significantly delayed and generally occurs when $E_{\text {thr }}$ is significantly below $E$. Then $e^{ \pm}$are created in high Landau states, and the absorption coefficient for the photon is given by $\kappa_{\text {abs }} \approx 4.3 \times 10^{7} b \exp \left(-8 m_{e} c^{2} / 3 b E \sin \vartheta\right) \mathrm{cm}^{-1}$ (Erber 1966).

The model described in this paper predicts that practically all photons that convert to $e^{ \pm}$do so in the region $b>0.1$, and very close to their emission points. Then the photon energy $E$ is shared by two particles created in the ground Landau state with energies $E / 2$.

\section{Splitting}

Following selection rules (Adler 1971; Usov 2002), we assume that only one splitting channel is allowed: $\perp \rightarrow\|+\|$ (Adler [1971] uses the opposite notation for the polarizations states, $\perp \leftrightarrow \|$ ). The absorption coefficient for a $\perp$ photon of energy $E$ propagating at an angle $\vartheta$ with respect to the magnetic field lines is given by

$$
\kappa_{\mathrm{sp}}=0.12 \zeta(b \sin \vartheta)^{6}\left(\frac{E}{m_{e} c^{2}}\right)^{5} \mathrm{~cm}^{-1}
$$

where $\zeta=1$ if $b \ll 1$ and $\zeta \sim 4 b^{-6}$ in the limit of $b \gg 1$ (the exact asymptotic value depends on $E$ sin $\vartheta$, see Baring $\&$ Harding 1997). Factor $\zeta$ can be calculated numerically (Figure 8 in Adler [1971] and Figure 1 in Baring \& Harding [1997]). Medin \& Lai (2010) and our Monte-Carlo simulations use an approximate formula,

$$
\zeta=(g+0.05)^{-1}(0.25 g+20)^{-1}, \quad g=b^{3} \exp \left[-0.6\left(\frac{E \sin \vartheta}{2 m_{e} c^{2}}\right)^{3}\right] .
$$

When a photon splits, its energy $E$ is shared between two daughter photons propagating in the same direction. The probability distribution for the energy a daughter photon $E_{1}$ is approximately given by $p(u) \approx 30 u^{2}(1-u)^{2}$, where $u=E_{1} / E$ (Baring \& Harding 1997). We use this distribution in our Monte-Carlo simulations.

\section{Implementation of splitting and conversion in Monte-Carlo simulations}

To determine the fate of high-energy photons generated by resonant scattering we follow the photon along its trajectory and track the change of its pitch angle $\vartheta$ with respect to the local magnetic field lines. The (initially small) angle grows due to the curvature of the field lines while the photon polarization remains unchanged along the trajectory (the small difference of the refraction indices for the two polarization states results in locking/adiabatic tracking of the photon polarization). For photons with $\|$ polarization, we check if they convert to $e^{ \pm}$. The threshold for conversion $E_{\mathrm{thr}}=2 m_{e} c^{2} / \sin \vartheta$ is reduced as $\vartheta$ increases. When $E_{\mathrm{thr}} / E$ is reduced below unity, conversion becomes possible, and in practice occurs immediately. For photons with $\perp$ polarization, we check if they split or convert to $e^{ \pm}$. In practice conversion is negligible as splitting occurs before the conversion threshold condition is satisfied.

Consider a photon emitted along the outflow direction at point $\mathbf{r}_{\mathrm{em}}$. Let $E_{\max }\left(\mathbf{r}_{\mathrm{em}}\right)$ be the maximum energy for the photon to avoid destruction and escape. More precisely, we define two maximum energies $E_{\mathrm{max}}^{\|}\left(\mathbf{r}_{\mathrm{em}}\right)$ and $E_{\mathrm{max}}^{\perp}\left(\mathbf{r}_{\mathrm{em}}\right)$ for $\|$ and $\perp$ photons, respectively. For any given magnetospheric configuration, $E_{\max }^{\|}\left(\mathbf{r}_{\mathrm{em}}\right)$ and $E_{\max }^{\perp}\left(\mathbf{r}_{\mathrm{em}}\right)$ can be calculated numerically by following the photon trajectories as described above.

The fate of an emitted photon in our Monte-Carlo simulations is determined by comparing its energy $E$ with $E_{\text {max }}$. The simulation can be further simplified due to the following observation: if a resonantly scattered photon is destroyed, the destruction happens quickly, close to the emission point $\mathbf{r}_{\text {em }}$ (except for a negligible number of photons scattered near the magnetic axis, where the field line curvature is small). This is because the high-energy photons capable of conversion or splitting are generated in the region of strong and curved magnetic field $b \gtrsim 0.1$ (recall that the energy of a resonantly scattered photon does not exceed $2 \gamma_{\mathrm{sc}} b m_{e} c^{2} \approx 200 b^{2} m_{e} c^{2}$ ). In the Monte-Carlo simulations it is a good approximation to assume the immediate conversion of $\|$ photons if $E>E_{\max }^{\|}\left(\mathbf{r}_{\mathrm{em}}\right)$ and the immediate splitting of $\perp$ photons if $E>E_{\max }^{\perp}\left(\mathbf{r}_{\mathrm{em}}\right)$. This approximation is used in all sample models shown in Section 4 .

Let $\bar{E}\left(\mathbf{r}_{\mathrm{em}}\right)$ be the average energy of resonantly scattered photons. Most of the photons generated in the zone $\bar{E}>E_{\max }$ are destroyed. As the outflow moves along a field line, there is a sharp transition to photon escape at the boundary $\mathbf{r}_{0}$ defined by $\bar{E}\left(\mathbf{r}_{0}\right)=E_{\max }\left(\mathbf{r}_{0}\right)$. Similar results will be obtained if one assumes that all photons scattered with $E<E_{\max }\left(\mathbf{r}_{0}\right)$ escape (regardless of their emission points $\mathbf{r}_{\mathrm{em}}$ ), and all photons with $E>E_{\text {max }}\left(\mathbf{r}_{0}\right.$ ) split or convert, depending on their polarization states. Such an approximation is used in the cascade simulations in Section 3.2. Instead of the pre-calculated $E_{\max }^{\|}\left(\mathbf{r}_{\mathrm{em}}\right)$ and $E_{\max }^{\perp}\left(\mathbf{r}_{\mathrm{em}}\right)$ (which depend on the chosen dipole magnetic configuration), we assume a given fixed $E_{\max }^{\|}=E_{\max }^{\perp} \approx \gamma_{\mathrm{sc}}\left(b_{0}\right) b_{0} m_{e} c^{2}$. The value of $E_{\max }$ is typically somewhat above $1 \mathrm{MeV}$, depending on the curvature of magnetic field lines. Simulations shown in Figures $3-6$ assume $E_{\text {max }}=3 m_{e} c^{2}$ - a typical value obtained with the more detailed simulations that follow the propagation of photons through the magnetosphere. 


\section{Boundary between the pair-loading and radiative zones}

The location of the boundary between the two zones was estimated in Section 2.3. Approximately, it is where the magnetic field $b=B / B_{Q}$ equals $b_{0} \sim 1 / 4$. The exact $b_{0}$ varies for different field lines of the magnetosphere. It is convenient to label the field lines by their maximum extension radius $R_{\max }$ (the apex radius). The boundary location also depends on the magnetic moment of the star $\hat{\mu}$ and its temperature $T$. These dependencies may be formulated once we specify a more rigorous definition of the boundary.

To define the radiative zone, consider a photon scattered at a given point $\mathrm{P}$ by an electron with Lorentz factor $\gamma_{\mathrm{sc}}$ (Equation 12). We focus on the region where $b \ll 1$ and $\gamma_{\mathrm{sc}} \gg 1$, as the estimated zone boundary is in this region. The energy of the scattered photon is given by $E=\gamma_{\mathrm{sc}}\left(1+\beta_{\mathrm{sc}} \cos \tilde{\vartheta}\right) b m_{e} c^{2}$ where $\tilde{\vartheta}$ is the scattering angle in the electron rest frame. The mean expectation for $\cos \tilde{\vartheta}$ is zero and the average photon energy after scattering is $\gamma_{\mathrm{sc}} b m_{e} c^{2}$. The photon direction in the lab frame is nearly aligned with the electron velocity, within an angle $\vartheta \approx \gamma_{\mathrm{sc}}^{-1}$, and hence the photon is emitted nearly parallel to the local magnetic field. The photon propagates along the straight line through the magnetosphere (the bending of photon trajectory by neutron-star gravity is neglected). We adopt the following definition: point $\mathrm{P}$ belongs to the radiative zone if a photon (in the $\|$ polarization state) emitted parallel to $\mathbf{B}$ with energy $\gamma_{\mathrm{sc}} b m_{e} c^{2}$ does not convert to a pair and escapes the magnetosphere.

We have numerically calculated the boundary of the radiative zone for dipole magnetospheres around neutron stars with various magnetic moments and temperatures. The boundary is a closed surface around the star, which may be described in spherical coordinates by a function $r_{0}(\theta)$. Instead of $r$ and $\theta$ we find it more convenient to use the coordinates $R_{\max }$ and $b$, where $R_{\max }$ labels the field lines and the value of $b$ is used as a coordinate running along the field line. Then the boundary is described by a function $b_{0}\left(R_{\max }\right)$. The radiative zone only exists on sufficiently extended field lines,

$$
R_{\max }>R_{0} \approx\left(\frac{\hat{\mu}}{B_{Q}}\right)^{1 / 3}\left(\frac{K}{2}\right)^{1 / 6} \approx 5.4 \times 10^{6}\left(\frac{\hat{\mu}}{10^{33} \mathrm{G} \mathrm{cm}^{3}}\right)^{1 / 3}\left(\frac{k T}{0.5 \mathrm{keV}}\right)^{-1 / 6} \mathrm{~cm}
$$

The numerically calculated $b_{0}$ for $R_{\max }>R_{0}$ is well approximated by

$$
b_{0}\left(R_{\max }\right)=0.265\left(\frac{\hat{\mu}}{10^{33} \mathrm{G} \mathrm{cm}^{3}}\right)^{0.15}\left(\frac{k T}{0.5 \mathrm{keV}}\right)^{0.39}\left(\frac{R_{\max }}{R_{\star}}-1\right)^{1 / 4}
$$

where

$$
R_{\star}=5 \times 10^{6}\left(\frac{\hat{\mu}}{10^{33} \mathrm{G} \mathrm{cm}^{3}}\right)^{0.31}\left(\frac{k T}{0.5 \mathrm{keV}}\right)^{-0.14} \mathrm{~cm} .
$$

The corresponding location of the boundary in spherical coordinates, $r_{0}(\theta)$, is determined by the relations $R_{\text {max }}=$ $r / \sin ^{2} \theta$ and $B=\left(\hat{\mu} / r^{3}\right)\left(1+3 \cos ^{2} \theta\right)^{1 / 2}$. The exact boundary location is reproduced by Equation (B4) with the accuracy of a few percent in a broad range of relevant $T(0.2<k T<1 \mathrm{keV})$ and $\hat{\mu}>3 \times 10^{31} \mathrm{G} \mathrm{cm}^{3}$. Note that $R_{\star}$ appearing in Equation (B4) is slightly smaller than $R_{0}$. For the models shown in this paper, $\hat{\mu}=5 \times 10^{32} \mathrm{G} \mathrm{cm}^{3}$, $k T=0.5 \mathrm{keV}$, and $R_{\star} \approx 0.9 R_{0}$.

We have also calculated the free path of scattered photons to absorption, $l_{\text {abs }}$, in the pair-loading zone $b>b_{0}$. It depends on the scattering location and the scattering angle. Practically all photons scattered in the zone $b>b_{0}$ have $l_{\text {abs }} / r \ll 1$, i.e. they immediately convert to $e^{ \pm}$pairs after scattering. At the boundary $b=b_{0}$, there is a steep transition to the escape regime $l_{\mathrm{abs}}=\infty$. The small $l_{\mathrm{abs}}$ in the pair-loading zone implies that photons scattered on a field line $R_{\max }$ convert to pairs on almost the same field line. For the sample models presented in this paper, we found that the typical shift in $R_{\max }$ before conversion is $\delta R_{\max } / R_{\max } \sim 0.1$ for photons scattered at $b=b_{0} ; \delta R_{\max }$ quickly decreases to essentially zero for photons scattered at $b>b_{0}$. This fact simplifies calculations in Section 3 , as one can assume that the processing of the outflow energy into $e^{ \pm}$pairs occurs independently on different field lines.

Note that $b_{0}$ significantly increases near the magnetic axis, where $u=R / R_{\max } \rightarrow 0$; sufficiently close to the axis, the pair loading zone disappears. This fact is, however, of little importance, as the maximum electric current, which corresponds to twist amplitude $\psi \sim 1$, is small near the axis $\left(I \propto u^{2}\right.$, see Equation [C7]), and its contribution to the observed luminosity is negligible. The luminosity peaks as $u^{2}$ at larger $u$; in this region $b_{0} \sim 1 / 4$.

\section{EMISSIVITY OF THE J-BUNDLE}

Section 3 described how the net emission from the j-bundle is obtained by integrating along thin magnetic tubes and then taking the sum over the tubes. It is also useful to have an explicit expression for the spatial distribution of emission (e.g. in spherical coordinates) for a concrete magnetospheric configuration. Below we write down the general expression for the emissivity per unit volume and then specialize to approximately dipole (moderately twisted) magnetospheres.

First note that a short piece $d l$ of a thin tube with magnetic flux $d \digamma$ has volume

$$
d V=\frac{d \digamma}{B} d l
$$


where we used the fact that the perpendicular cross section of the flux tube equals $B^{-1} d \digamma$. Then, using $d L / d \digamma=$ $\chi \Phi d I / d \digamma$ (Equation 27) and $d L / d \digamma d b=b_{0}^{-1} d L / d \digamma$ (Equation 24), we find the produced luminosity per unit volume

$$
\frac{d L}{d V}=\frac{\chi \Phi}{2 b_{0}}\left|\frac{d I}{d \digamma} \frac{d B}{d l}\right|
$$

Here the factor of $1 / 2$ takes into account that $d b$ corresponds to two pieces $d l$ of the tube, as the tube has two "legs" (two footpoints); $\Phi$ is the voltage between the footpoints, i.e. the net voltage along the tube. Equation (C2) shows that $d L / d V$ depends on the distribution of electric current among the magnetic field lines in the j-bundle; this distribution is given by

$$
\frac{d I}{d \digamma}=\frac{j}{B} \approx \frac{c \psi}{4 \pi R_{\max }},
$$

where $j$ is the current density. In Equation (C3) we used the Maxwell equation $\nabla \times \mathbf{B}=(4 \pi / c) \mathbf{j}$ with neglected displacement current, and substituted $\nabla \times \mathbf{B} \approx \psi B / R_{\max }$. This relation is derived below for the twisted dipole magnetosphere; it remains approximately valid for more general configurations. Substitution of Equation (C3) to Equation (C2) gives

$$
\frac{d L}{d V}=\frac{\chi \Phi}{b_{0}}\left|\frac{d B}{d l}\right| \frac{c \psi}{8 \pi R_{\max }},
$$

where $R_{\max }$ is the apex radius of the magnetic field line passing through $d V$.

Consider a dipole magnetosphere with a moderate axisymmetric twist. Its magnetic field is well approximated as the sum of a dipole poloidal field and a toroidal field $B_{\phi}$ (see B09). The poloidal flux function is that of a normal dipole,

$$
\digamma(r, \theta)=2 \pi \hat{\mu} \frac{\sin ^{2} \theta}{r}=\frac{2 \pi \hat{\mu}}{R_{\max }} .
$$

It is also convenient to use the dimensionless flux function

$$
u=\frac{\digamma R}{2 \pi \hat{\mu}}=\frac{R}{R_{\max }},
$$

where $R$ is the radius of the neutron star and $R_{\max }$ is the apex radius of the magnetic field line. Variable $u<1$, like $\digamma$, can be used to label the magnetic flux surfaces or magnetic field lines in the axisymmetric magnetosphere. Suppose the region $u<u_{\star}$ is activated, i.e. carries electric currents, and has approximately uniform twist amplitude $\psi \approx$ const. The electric current flowing between the magnetic axis and a flux surface $u$ is given by (B09),

$$
I(u) \approx \frac{c \hat{\mu} \psi u^{2}}{4 R^{2}} .
$$

It gives $d I / d u$ and $d I / d \digamma$ that confirms Equation (C3). One can also derive an explicit expression for $d B / d l$ that appears in Equation (C2). For the approximately dipole field, $B=\hat{\mu} r^{-3}\left(1+3 \cos ^{2} \theta\right)^{1 / 2}$ and

$$
\frac{d B}{d l}=-\frac{3 \hat{\mu}\left(3+5 \cos ^{2} \theta\right) \cos \theta}{r^{4}\left(1+4 \cos ^{2} \theta\right)} \text {. }
$$

\section{REFERENCES}

Adler, S. L. 1971, Ann. Phys., 67, 599

Baring, M. G., \& Harding, A. K. 1997, ApJ, 482, 372

Baring, M. G., \& Harding, A. K. 2007, Ap\&SS, 308, 109

Beloborodov, A. M. 2009, ApJ, 703, 1044 (B09)

Beloborodov, A. M. 2011, in High-Energy Emission from Pulsars and their Systems, Astrophysics and Space Science Proceedings, 299 (Springer-Verlag), arXiv:1008.4388

Beloborodov, A. M., \& Thompson, C. 2007, ApJ, 657, 967

Bhatia, V. B., Chopra, N., \& Panchapakesan, N. 1988, Ap\&SS, 150, 181

Canuto, V., Lodenquai, J., \& Ruderman, M. 1971, Phys. Rev. D, 3, 2303

den Hartog, P. R., Kuiper, L., \& Hermsen, W. 2008a, A\&A, 489, 263

den Hartog, P. R., Kuiper, L., Hermsen, W., Kaspi, V. M., Dib, R., Knödlseder, J., \& Gavriil, F. P. 2008b, A\&A, 489, 245

Enoto, T., Nakazawa, K., Makishima, K., Rea, N., Hurley, K., \& Shibata, S. 2010, ApJ, 722, L162

Erber, T. 1966, Rev. Mod. Phys., 38, 626

Fernández, R., \& Davis, S. W. 2011, ApJ, 730, 131

Fernández, R., \& Thompson, C. 2007, ApJ, 660, 615

Harding, A. K., \& Daugherty, J. K. 1991, ApJ, 374, 687

Heyl, J. S., \& Hernquist, L. 2005, MNRAS, 362, 777

Kaspi, V. M., \& Boydstun, K. 2010, 710, L115

Kuiper, L., Hermsen, W., \& Mendez, M. 2004, ApJ, 613, 1173

Kuiper, L., den Hartog, P. R., \& Hermsen, W. 2008 (arXiv:0810.4801)

Lyubarsky, Y., \& Eichler, D. 2007 (arXiv:0706.3578) 
Medin, Z., \& Lai, D. 2010, MNRAS, 406, 1379

Nobili, L., Turolla, R., \& Zane, S. 2008, MNRAS, 386, 1527

Parfrey, K., Beloborodov, A. M, \& Hui, L. 2012a, 754, L12

Parfrey, K., Beloborodov, A. M, \& Hui, L. 2012b, ApJ, submitted

Rea, N., Zane, S., Turolla, R., Lyutikov, M., \& Götz, D. 2008, ApJ, 686, 1245

Thompson, C., \& Beloborodov, A. M. 2005, ApJ, 634, 565

Thompson, C., Duncan, R. C., Woods, P. M., Kouveliotou, C., Finger, M. H., \& van Paradijs, J. 2000, ApJ, 543, 340

Thompson, C., Lyutikov, M., \& Kulkarni, S. R. 2002, ApJ, 574, 332

Shabad, A. E., \& Usov, V. V. 1986, Ap\&SS, 128, 377

Usov, V. V. 2002, ApJ, 572, L87

Ventura, J. 1979, Phys. Rev. D, 19, 1684

Wolfson, R. 1995, ApJ, 443, 810

Woods, P. M., \& Thompson, C. 2006, in Compact Stellar X-Ray Sources, ed. W. H. G. Lewin \& M. van der Klis (Cambridge: Cambridge Univ. Press), 547 